\title{
A semi-Lagrangian scheme for mean curvature motion with nonlinear Neumann conditions
}

\author{
YVES ACHDOU
}

Univ. Paris Diderot, Sorbonne Paris Cité, Laboratoire Jacques-Louis Lions, UMR 7598, UPMC, CNRS, 75205 Paris, France

E-mail: achdou@ljll.univ-paris-diderot.fr

\section{MAURIZIO FALCONE}

Dipartimento di Matematica, Università Roma “La Sapienza”, Piazzale A. Moro 2, 00185 Roma

E-mail:falcone@mat.uniroma1.it

[Received 12 January 2011 and in revised form 11 March 2012]

\begin{abstract}
A numerical method for mean curvature motion in bounded domains with nonlinear Neumann boundary conditions is proposed and analyzed. It consists of a semi-Lagrangian scheme in the main part of the domain as proposed by Carlini, Falcone and Ferretti, combined with a finite difference scheme in small layers near the boundary to cope with the boundary condition. The consistency of the new scheme is proved for nonstructured triangular meshes in dimension two. The monotonicity of a regularized version of the scheme with some additional vanishing artificial viscosity is studied. Details on the implementation are given. Numerical tests are presented.
\end{abstract}

2010 Mathematics Subject Classification: Primary 65M06, 65M12, 49L25.

Keywords: Hamilton Jacobi equations, front propagation, Neumann boundary conditions, convergence, viscosity solutions, triangular meshes.

\section{Introduction}

We consider the mean curvature equation

$$
\frac{\partial u}{\partial t}-\operatorname{trace}\left(\left(I-\frac{D u \otimes D u}{|D u|^{2}}\right) D^{2} u\right)=0, \quad \text { in } \Omega \times(0, T),
$$

where $\Omega$ is a bounded domain of $\mathbb{R}^{n}$ with a $W^{3, \infty}$ boundary. The partial differential equation (1) can also be written

$$
\frac{\partial u}{\partial t}-\Delta u+\frac{\left(D^{2} u D u, D u\right)}{|D u|^{2}}=0, \quad \text { in } \Omega \times(0, T),
$$

or

$$
\frac{\partial u}{\partial t}-\operatorname{div}\left(\frac{D u}{|D u|}\right)|D u|=0, \quad \text { in } \Omega \times(0, T) .
$$

Here, $D u$ stands for the space gradient, $D^{2} u$ for the Hessian matrix of second space derivatives of $u$ and for any vector $p \in \mathbb{R}^{n}, p \otimes p=p p^{T}$. The partial differential equation (1) is complemented with an initial condition

$$
u(x, 0)=u_{0}(x), \quad \text { for } x \in \Omega,
$$


and with the nonlinear Neumann condition

$$
\frac{\partial u}{\partial n}=\theta|D u| \quad \text { on } \quad \partial \Omega \times(0, T),
$$

where $\theta$ is a Lipschitz continuous function with $|\theta(x)| \leqslant \bar{\theta}<1$.

To put our work into perspective, let us recall that Crandall and Lions [13] have developed an analysis of explicit finite difference schemes for a class of possibly degenerate parabolic equations of the form:

$$
u_{t}-\operatorname{trace}\left(\Theta(x, D u) \Theta(x, D u)^{T} D^{2} u\right)=0 \quad \text { for } t>0, x \in \mathbb{R}^{n} .
$$

Here, $\Theta(x, p)$ is a $n \times m$ matrix valued function of $(x, p) \in \mathbb{R}^{n} \times \mathbb{R}^{n}$ and $A^{T}$ denotes the transpose of a matrix $A$. Note that the integer $m$ is arbitrary and the equation is degenerate if $\Theta(x, p)$ is not invertible.

Our particular case (1) corresponds to the choice

$$
\Theta(x, p)=\Theta(p)=I-\frac{p \otimes p}{|p|^{2}},
$$

(where $|\cdot|$ denotes the Euclidean norm). It is well known that in our case $\Theta$ is discontinuous at $p=0$ and degenerate for $p \neq 0$. Since $\Theta(p)^{2}=\Theta(p)$ and $\Theta(p)=\Theta(p)^{T}, \Theta(p)$ is a projection matrix, which in fact projects the diffusion orthogonally with respect to the gradient.

We will work in the framework of level set methods using the theory of viscosity solutions, which allows to deal with both singularities of solutions and degeneracies of the parabolic operator (see [2] and [11] for an introduction to this theory). Existence and uniqueness for the viscosity solutions to the Cauchy problem in $\mathbb{R}^{n}$ have been proved independently by Evans and Spruck [16] and by Chen, Giga and Goto [9]. Starting from those pioneering papers, the last years have witnessed a great development of the theory about curvature related flows, as well as its application to various fields like phase transitions, image processing, fluid dynamics, material science and crystallography (see the books [28] and [25] for a review of interesting applications and simulations). We refer the interested reader to the lecture notes [19], [31] for the theory of viscosity solutions for surface evolution equations. Viscosity solutions of the boundary value problem (1), (4), (5) have been studied by Barles [3], see also Ishii and Ishii [21], Giga et al. [20].

Naturally, several attempts have been made to construct reliable approximation schemes. In particular, Evans [15] and Barles-Georgelin [4] proved the convergence of semi-discrete approximations (only time is discretized). Finite difference schemes have been proposed by Osher and Sethian in the late eighties, see [26] but the proof of convergence results for fully discrete approximation schemes came later with the papers by Catté, Dibos and Koepfler [8], Crandall and Lions [13] and Oberman [24]. Let us also mention that the approximation of the Mean Curvature Flow has been also tackled via finite element methods by several authors, although this approach usually suffers from the degeneracy of the second order operator. The main convergence results related to this approach which can take into account also the onset of singularities can be found in the papers by Nochetto and Verdi [23] and Dziuk and Deckelnick [14].

In this work, we aim at modifying the semi-Lagrangian scheme introduced by Carlini et al in the recent article [7] for (1) with no boundary conditions. The idea is to combine the previously mentioned scheme with a finite difference scheme for (5) in thin layers near $\partial \Omega$. Note that a first version of the semi-Lagrangian scheme had been introduced in [18] where consistency was proved 
assuming that the gradient of the solution could not vanish, i.e., in the nonsingular case. The second version proposed in [7] can handle the singular case. For the latter, consistency, monotonicity (in a generalized sense), and thus convergence were proved. The main ingredient of the proof was a generalization of the result obtained for monotone schemes by Barles and Souganidis in [5]. In particular, this generalization allows for relaxing the strong monotonicity condition which is usually required and to prove convergence for a regularized scheme (see Section 2 in [7] for more details about this relaxation).

In [7], the semi-Lagrangian scheme has only been defined for uniform Cartesian grids. Since we wish to deal with general domains, we have decided to use triangular meshes adapting the regularized scheme analyzed in [7]. Hence we need two more ingredients: the use of triangular meshes to accurately approximate the geometry and a local solver in small regions near $\partial \Omega$ for a first order Hamilton-Jacobi equation corresponding to the Neumann boundary condition. We will focus on the two-dimensional case (i.e., $n=2$ ), for simplicity and because it is possible to obtain a convergence result in this case, but the scheme presented here can be used for three dimensional problems with tetrahedral meshes. We should mention that relatively few numerical schemes for Hamilton-Jacobi equation on nonstructured meshes have been proposed in the literature, probably because the hyperbolic nature of these equations and the link between viscosity solutions and entropy solutions for conservation laws have supported the use of structured grids and finite difference schemes: we mention the book of Sethian [28], and [1] in which Abgrall has proved convergence for a scheme based on triangular meshes for Hamilton-Jacobi equations of the first order. Note that semi-Lagrangian schemes in the class proposed by Falcone and Ferretti in [17] do not require structured grids and can be applied also to stationary problems. For example, in [27] a first order semi-Lagrangian method on unstructured grids is used to solve the first order HamiltonJacobi equation corresponding to the Shape-from-Shading problem. However, we preferred to use for the implementation of the boundary condition (5) a Godunov type scheme on the triangular mesh, and, to be more precise, an adaptation of the fast sweeping method for the eikonal equation on structured meshes originally proposed by Zhao [32].

We will extend some of the theoretical results contained in [7] to deal with the case of an open bounded domain $\Omega$ with Neumann boundary conditions. The general framework for our convergence result is the theory of viscosity solutions and, in particular, the results that have been established by Barles [3] (see also Ishii and Ishii [21]).

The paper is organized as follows: In Section 2 we introduce our notations, present the scheme and give some hints for the implementation. Section 3 is devoted to the analysis of consistency and monotonicity; these two basic properties are necessary to establish convergence via the generalization of Barles-Souganidis abstract theorem proved in [7]. In particular, in $\S 3.1$, we prove that the scheme is consistent. For the monotonicity, we actually regularize the proposed scheme by the addition of vanishing artificial viscosity. In $\S 3.2$, we present an analysis of the monotonicity for a regularization based on the addition of a viscosity term, as well as a convergence result for this regularized version of the scheme. In Section 4, we report about some numerical results, obtained with the proposed scheme, i.e., without the above mentioned regularization.

\section{A numerical method in dimension two}

An ingredient of the numerical method is the semi-Lagrangian scheme proposed in [7] on a triangular mesh. The semi-Lagrangian method consists essentially of applying a finite difference scheme along a characteristic curve. The spatial step used in the semi-Lagrangian method will be of 


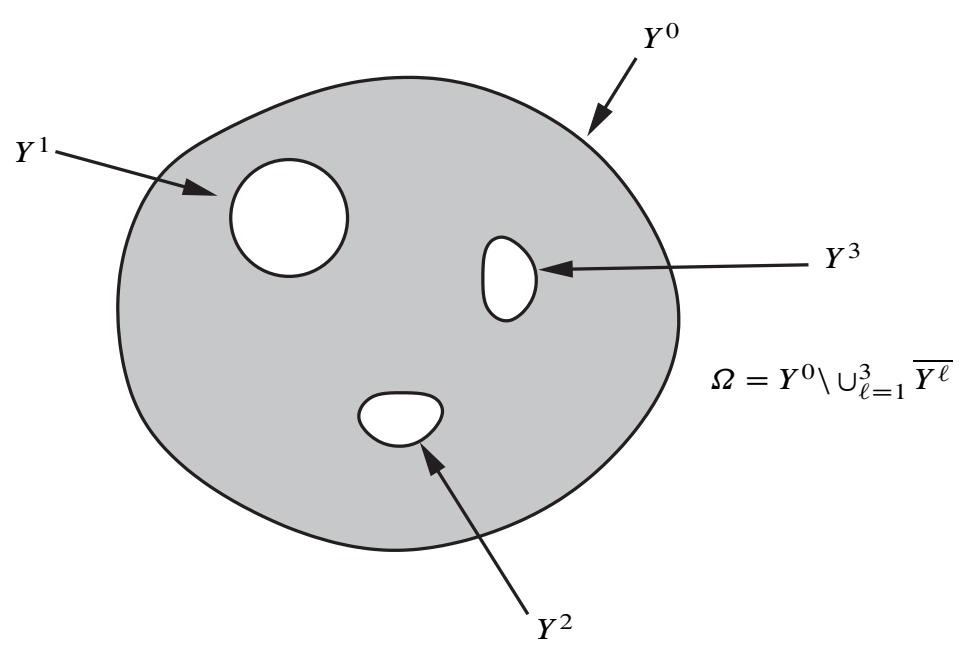

FIG. 1. The domains $\left(Y^{\ell}\right)_{\ell=0, \ldots, 3}$

the order of $\delta$. Therefore, for a node $\xi$ whose distance to the boundary is smaller than $\delta$, it is possible that the points needed by the scheme (the feet of the characteristics) fall out of the domain $\Omega$. This is the essential reason why we choose to distinguish thin layers near the boundary (whose width is of the order of $\delta$ ) in which we will use a finite difference scheme for the boundary condition (5) rather than (1).

In $\S 2.1$, we start by carefully defining the geometry, the triangular mesh and the thin layers near the boundary. We recall some basic facts on finite elements on triangular meshes in $\S 2.2$, and we present the scheme in $\S 2.3$.

\subsection{A nonstructured mesh}

2.1.1 The domain $\Omega$. Consider $\left(Y^{\ell}\right)_{\ell=0, \ldots, L}, L+1$ simply connected bounded domains of $\mathbb{R}^{2}$ such that $Y^{\ell} \subset \subset Y^{0}$ if $1 \leqslant \ell \leqslant L$ and $\overline{Y^{\ell}} \cap \overline{Y^{j}}=\emptyset$ if $1 \leqslant j<\ell \leqslant L$, see Figure 1 . The sets $\overline{Y^{\ell}}$, $1 \leqslant \ell \leqslant L$ will be the holes of the domain $\Omega$ defined below.

Call $\left(\Gamma^{\ell}\right)_{\ell=0, \ldots, L}$ the boundaries of $\left(Y^{\ell}\right)_{\ell=0, \ldots, L}$. The curves $\Gamma^{\ell}$ are closed, connected and disjoint. We assume that $\Gamma^{\ell}$ is parameterized by $\left(\phi_{1}^{\ell}(\theta), \phi_{2}^{\ell}(\theta)\right.$ where $\phi_{1}^{\ell}$ and $\phi_{2}^{\ell}$ are two smooth real valued functions defined in $\mathbb{T}$ the unit one-dimensional torus. We assume that $\left(\phi_{1}^{0}(\theta), \phi_{2}^{0}(\theta)\right)$ turns counterclockwise as $\theta$ grows from 0 to 1 , and that for $1 \leqslant \ell \leqslant L,\left(\phi_{1}^{\ell}(\theta), \phi_{2}^{\ell}(\theta)\right)$ turns clockwise as $\theta$ grows from 0 to 1 .

Call $\Omega=Y^{0} \backslash \cup_{\ell=1}^{L} \overline{Y^{\ell}}$. We have $\partial \Omega=\cup_{\ell=0}^{L} \Gamma^{\ell}$.

The curvilinear abscissa $s^{\ell}$ is given by

$$
s^{\ell}(0)=0 \quad \text { and } \quad \frac{d s^{\ell}}{d \theta}(\theta)=\sqrt{\left(\frac{d \phi_{1}^{\ell}}{d \theta}(\theta)\right)^{2}+\left(\frac{d \phi_{2}^{\ell}}{d \theta}(\theta)\right)^{2}} .
$$

Call $S^{\ell}=s^{\ell}(1)$. There exist two smooth $S^{\ell}$-periodic real valued functions $x_{1}^{\ell}$ and $x_{2}^{\ell}$ such that 
$s \rightarrow\left(x_{1}^{\ell}(s), x_{2}^{\ell}(s)\right)$ is a parametrization of $\Gamma^{\ell}$. The exterior normal to $\partial \Omega$ at $\left(x_{1}^{\ell}(s), x_{2}^{\ell}(s)\right)$ is $n(s)=\left(\frac{d x_{2}^{\ell}}{d s}(s),-\frac{d x_{1}^{\ell}}{d s}(s)\right)$.

2.1.2 The mesh. Let us consider a family $\left(\mathcal{J}_{h}\right)$ of regular and quasiuniform triangular meshes of $\Omega$. We denote by $N_{h}$ the number of nodes of $\tau_{h}$. More precisely, $\tau_{h}$, is a set of triangles such that

- all the nodes lie in $\bar{\Omega}$.

- the intersection of two distinct triangles is either empty or a common vertex or a whole common edge of both triangles.

- the diameters of any triangle in $\tau_{h}$ and of its inscribed circle are both of the order of $h$.

- We define the open set $\Omega_{h}$ such that $\overline{\Omega_{h}}=\bigcup_{\tau \in \tau_{h}} \tau$ and we call $\left(\xi_{i}\right)_{1 \leqslant i \leqslant N_{h}}$ the nodes of $\mathcal{T}_{h}$. We assume that for all $i, 1 \leqslant i \leqslant N_{h}, \xi_{i} \in \partial \Omega_{h}$ if and only if $\xi_{i} \in \partial \Omega$, that for each $\ell=0, \ldots, L$, there are nodes of $\mathcal{J}_{h}$ lying on $\Gamma^{\ell}$, and that the Hausdorff distance between $\partial \Omega$ and $\partial \Omega_{h}$ is of the order of $h^{2}$.

- We assume that $\partial \Omega_{h}=\cup_{\ell=0}^{L} \Gamma_{h}^{\ell}$ where $\Gamma_{h}^{\ell}$ is a non empty polygonal line whose vertices are the nodes of $\tau_{h}$ lying on $\Gamma^{\ell}$.

2.1.3 Thin layers near $\partial \Omega$. Consider another positive number $\delta$ such that $\delta / h>N$, where $N$ is fixed positive integer. Let $\omega^{\ell}$ be the ring shaped domain defined by

$$
\overline{\omega^{\ell}}:=\left\{x \in \bar{\Omega}_{h}, d\left(x, \Gamma^{\ell}\right) \leqslant \delta+h\right\} .
$$

Since the thickness of $\omega^{\ell}$ is of the order of $\delta, \omega^{\ell} \subset \Omega_{h}$ and $\overline{\omega^{\ell}} \cap \overline{\omega^{k}}=\emptyset, k \neq \ell$ if $\delta$ is small enough. Hereafter, we will take $h$ and $\delta$ small enough so that all the geometrical notions can be defined without ambiguity.

The parameter $\delta$ will be the step used in the semi-Lagrangian scheme, in which, for a node $\xi$, one needs to evaluate a function at two points located at a distance $\delta$ of $\xi$ on an approximate characteristic curve. In $\omega^{\ell}$, this scheme cannot be applied, so we use a finite difference scheme for (5) instead.

DEFINITION 1 (Internal and boundary nodes) We say that a vertex $\xi$ of $\tau_{h}$ is a strongly internal mesh node if $\xi$ does not belong to $\cup_{\ell=0}^{L} \overline{\omega^{\ell}}$. In the opposite case, we say that $\xi$ is a boundary node.

Since the Hausdorff distance between $\partial \Omega$ and $\partial \Omega_{h}$ is of the order of $h^{2}$, if $\delta$ is small enough, then for all the strongly internal mesh nodes $\xi$,

$$
B(\xi, \delta) \subset \overline{\Omega_{h}} .
$$

DEFINITION 2 We define the set of nodes $\Xi_{h}^{\ell}$ by

$$
\Xi_{h}^{\ell}:=\left\{\xi ; \xi \text { is a boundary node lying in } \overline{\omega^{\ell}}\right\} .
$$

If $\xi$ is a boundary node, then there exists a unique $\ell, 0 \leqslant \ell \leqslant L$ such that $\xi \in \Xi_{h}^{\ell}$.

Hereafter, we assume that $\delta$ is small enough such that for any $\xi \in \Xi_{h}^{\ell}$, the projection $\pi^{\ell}(\xi)$ of $\xi$ on $\Gamma^{\ell}$ is uniquely defined as the point on $\Gamma^{\ell}$ which minimizes the distance to $\xi$. The distance of $\xi$ to $\Gamma^{\ell}$ is then $d\left(\xi, \Gamma^{\ell}\right)=\left|\xi-\pi^{\ell}(\xi)\right|$. Similarly, we can extend the definition of the normal vector to $\Gamma^{\ell}$ to the nodes in $\Xi_{h}^{\ell}$ by : for all $\xi \in \Xi_{h}^{\ell}, n^{\ell}(\xi)=n^{\ell}(\pi(\xi))$. We can also define $s^{\ell}(\xi)$ as the curvilinear abscissa of $\pi^{\ell}(\xi)$. 
In practice, the set of nodes $\Xi_{h}^{\ell}$ and then $\pi^{\ell}(\xi)$ for $\xi \in \Xi_{h}^{\ell}$ can be obtained in different ways. Without going to far into details, one can

- either solve eikonal equations on a much finer grid (a very fine uniform grid for example).

- or construct triangulations of regions approximating $\left\{x \in \Omega, d\left(x, \Gamma^{\ell}\right) \leqslant \delta+h\right\}$ with a high resolution in the tangential direction to $\Gamma^{\ell}$, and perform a search method to see if a node $\xi$ of $\widetilde{J}_{h}$ belongs to $\Xi_{h}^{\ell}$, and if yes to compute $\pi^{\ell}(\xi)$ (by dichotomy for instance).

The pair $\left(d\left(\xi, \Gamma^{\ell}\right), s^{\ell}(\xi)\right)$ is closed to an orthogonal system of coordinates in $\overline{\omega^{\ell}}$, and the nodes $\xi \in \Xi_{h}^{\ell}$ can be sorted by ordering either $\left(d\left(\xi, \Gamma^{\ell}\right), s^{\ell}(\xi)\right)$ or $\left(s^{\ell}(\xi), d\left(\xi, \Gamma^{\ell}\right)\right)$ lexicographically. These two kinds of indexing will prove useful for what follows.

As an example, assume that the domain $\Omega$ has a hole, so $L=1$; its outer boundary is parametrized by

$$
x_{1}=2 \cos (2 \pi t)+0.75 \cos (4 \pi t), \quad x_{2}=2 \sin (2 \pi t)+0.75 \sin (4 \pi t),
$$

and its inner boundary is the unit circle of equation $|x-(0.5,0)|=1$. We take $h \sim 0.06$ and $\delta \sim 0.3$. The mesh and the two layers near the boundaries are displayed in Figure 2: there are two lines around the layers' boundaries. Note that we have chosen a large value of $\delta$ for the layers to be visible.

\subsection{Piecewise linear finite elements}

For what follows, we need to define the space of functions $V_{h}=\left\{v \in \mathrm{C}^{0}\left(\overline{\Omega_{h}}\right),\left.v\right|_{\tau}\right.$ is affine, $\forall \tau \in$ $\left.\widetilde{J}_{h}\right\}$. For $i=1, \ldots, N_{h}$, let $\lambda_{i} \in V_{h}$ be the nodal basis function associated to node $\xi_{i}$, i.e., $\lambda_{i}\left(\xi_{j}\right)=$ $\delta_{i, j}, \forall j=1, \ldots, N_{h}$.

The finite element method for the heat equation in $\Omega_{h}$ with Neumann conditions on $\partial \Omega_{h}$ involves the stiffness matrix $A \in \mathbb{R}^{N_{h} \times N_{h}}$ and the mass matrix $M \in \mathbb{R}^{N_{h} \times N_{h}}$, which will be useful below:

$$
A_{i, j}=\int_{\Omega_{h}} D \lambda_{i}(x) \cdot D \lambda_{j}(x) d x, \quad M_{i, j}=\int_{\Omega_{h}} \lambda_{i}(x) \lambda_{j}(x) d x .
$$

It is well known that if all the triangles containing a vertex $\xi_{i}$ have acute angles then $A_{i, j} \leqslant 0$, for all $j \neq i$. Similarly, if all the triangles in a mesh $\widetilde{J}_{h}$ have all acute angles, then the resulting matrix $A$ is a M-matrix, (there is a discrete maximum principle).

We will sometimes make the following assumption:

ASSUMPTION 1 For all the strongly internal nodes $\xi_{i}$, all the triangles containing $\xi_{i}$ have acute angles, uniformly bounded away from 0 and $\pi / 2$. In such a case, if $\xi_{i} \neq \xi_{j}$ are two vertices of a triangle $t \in \mathcal{J}_{h}$ containing a strongly internal node, then

$$
A_{i, j} \leqslant-\alpha,
$$

for a positive constant $\alpha$ uniform w.r.t. $t$.

REMARK 1 In dimension two, if $\partial \Omega$ is smooth, it is always possible to construct such families of triangulations, by adding so-called Steiner nodes to the triangulation if necessary.

For what follows, we will need the following definitions: 


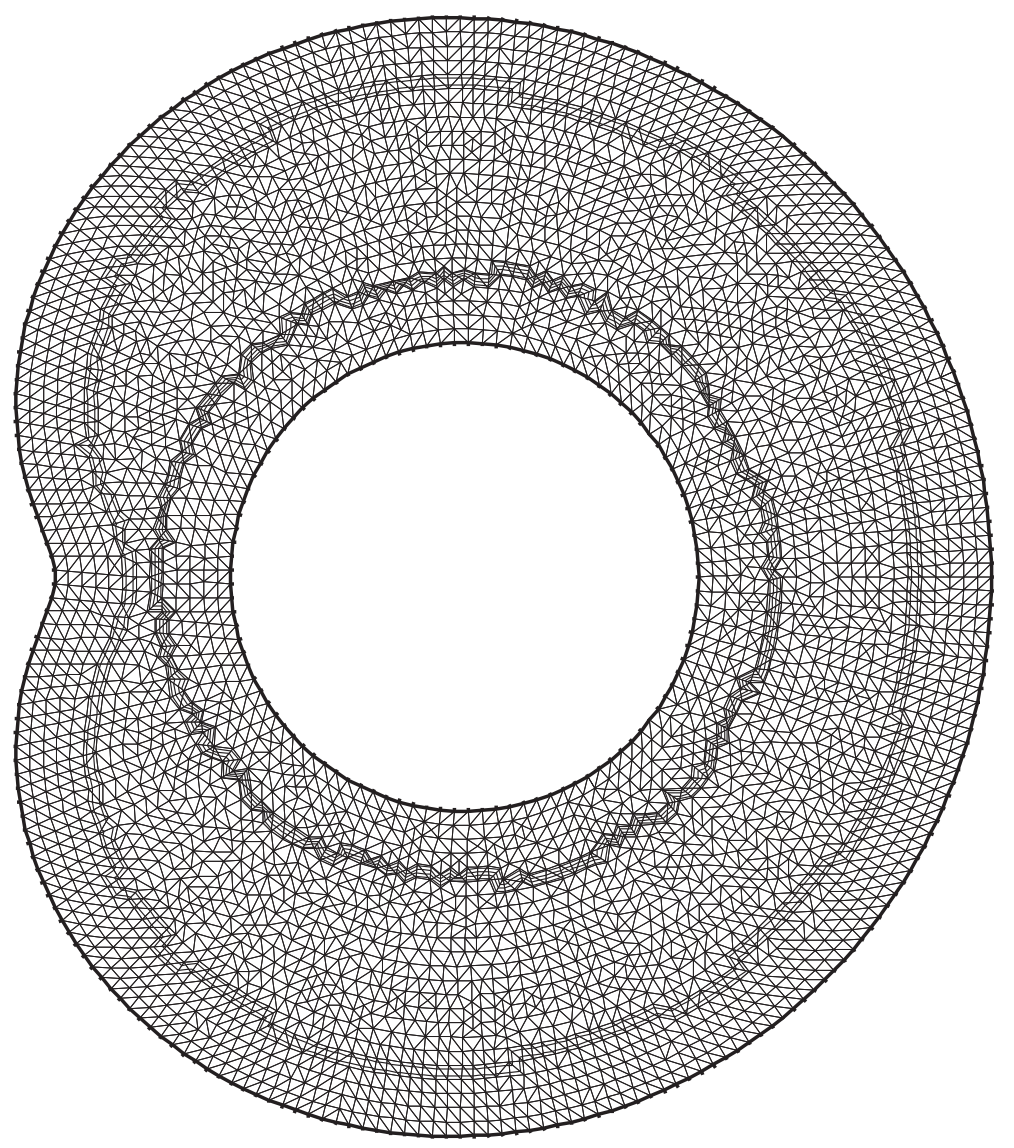

FIG. 2. The domain $\Omega$, the mesh and the two layers near the boundaries

- For a real valued function $w$ defined at the nodes of $\mathcal{J}_{h}$, we call $\ell_{h}[w]$ the piecewise affine interpolation of $w$.

- For a mesh node $\xi_{i}$, let $\mathcal{J}_{h, i}$ be the set of triangles $\tau$ of $\mathcal{J}_{h}$ such that $\xi_{i}$ is a vertex of $\tau$, and $\omega_{\xi_{i}}$ be the polygonal domain obtained as the union of the triangles in $\mathcal{J}_{h, i}$. We also define $\Sigma_{i}=\{j$ : $\xi_{j}$ is a vertex of $\left.\omega_{\xi_{i}}\right\}$.

\subsection{The scheme}

We approximate $u\left(\xi_{i}, n \Delta t\right), 1 \leqslant i \leqslant N_{h}$ by $u_{i}^{n}$ computed by a discrete numerical scheme. The proposed schemes differ according to the previously defined regions. At the strongly internal nodes, we will use the semi-Lagrangian scheme advocated by Carlini et al [7] whereas in the thin regions near $\partial \Omega$, namely $\overline{\omega^{\ell}}, \ell=0, \ldots, L$, we will use a finite difference scheme for the equation

$$
n^{\ell}(x) \cdot D u(x)-\theta|D u(x)|=0 \text {. }
$$

Note that the width of that region is controlled by the parameters $\delta$ and $h$ according to (8). 
2.3.1 The scheme in $\overline{\omega^{\ell}}, \ell=0, \ldots, L$. The nonlinear Neumann condition (12) is not only imposed at the nodes on $\partial \Omega^{h}$, but also at all the boundary nodes in $\overline{\omega^{\ell}}$. Consider the steady state Hamilton-Jacobi equation (12), and a monotone and consistent scheme for (12) at the nodes in $\Gamma_{h}^{\ell}$ which we write

$$
\mathbb{B}^{\ell}\left(\xi_{i}, u_{i},[u]_{\ell},[[u]]\right)=0, \quad \text { for all } i \text { such that } \xi_{i} \in \Xi_{h}^{\ell}
$$

where

$$
\begin{aligned}
{[u]_{\ell} } & =\left\{u_{j}, 1 \leqslant j \leqslant N_{h}, j \neq i, \xi_{j} \in \Xi_{h}^{\ell}\right\}, \\
{[[u]] } & =\left\{u_{j}, 1 \leqslant j \leqslant N_{h}, \xi_{j} \text { is strongly internal }\right\} .
\end{aligned}
$$

For example, a first order Godunov like scheme can be used, see $\S 2.4$. Note that such an upwind scheme written at a given node $\xi \in \Xi_{h}^{\ell}$ may involve values at strongly internal mesh nodes.

The values $u_{i}^{n+1}$, for $i$ such that $\xi_{i} \in \Xi_{h}^{\ell}$ are computed by solving the system of nonlinear equation

$$
\mathrm{B}^{\ell}\left(\xi_{i}, u_{i}^{n+1},\left[u^{n+1}\right]_{\ell},\left[\left[u^{n}\right]\right]\right)=0, \quad \text { for all } i \text { such that } \xi_{i} \in \Xi_{h}^{\ell} \text {. }
$$

The monotonicity of the scheme implies the existence and uniqueness of a solution, see [10].

System (14) is solved by a fast sweeping method as in [32], which consists of combining several Gauss-Seidel methods corresponding to different orderings of the nodes in $\Xi_{h}^{\ell}$ introduced in Definition 2; this is why it is useful to have several lexicographic orderings of the nodes in $\Xi_{h}^{\ell}$, all related to a close to orthogonal systems of coordinates (see $\S 2.1$ and $\S 2.4$ for more details on the implementation).

2.3.2 The scheme at the strongly internal nodes. At the strongly internal nodes, we essentially use the scheme proposed by Carlini et al [7], with a slight modification due to the unstructured character of the present mesh.

An important ingredient of the scheme is a linear discrete gradient operator: let $\xi_{i}$ be an internal mesh node. We assume that if $v$ is constant, then $D_{h} v=0$ and that the order of the approximation is $q>0$, i.e., if $\Phi$ is a smooth function, then

$$
\max _{i}\left|\left[D_{h} \Phi\right]\left(\xi_{i}\right)-D \Phi\left(\xi_{i}\right)\right| \leqslant C h^{q} .
$$

We also assume that for any function $v$ whose values at the mesh nodes is known, the discrete gradient of $v$ at $\xi_{i}$ is of the form

$$
\left[D_{h} v\right]\left(\xi_{i}\right)=\left(\begin{array}{c}
\sum_{j \in \Sigma_{i}} d_{i, 1}^{j} v\left(\xi_{j}\right) \\
\sum_{j \in \Sigma_{i}} d_{i, 2}^{j} v\left(\xi_{j}\right)
\end{array}\right),
$$

where the coefficients $d_{i, 1}^{j}$ and $d_{i, 2}^{j}$ do not depend on $v$, but only on the mesh. Let us give a possible construction of $D_{h}$.

Example Let $v$ be a continuous function defined on $\Omega_{h}$, such that for all $\tau \in \mathcal{J}_{h},\left.v\right|_{\tau}$ is affine. Define the discrete gradient $\left[D_{h} v\right]\left(\xi_{i}\right)$ by

$$
\left[D_{h} v\right]\left(\xi_{i}\right)=\sum_{\tau \in \mathcal{J}_{h, i}} \frac{|\tau|}{\left|\omega_{\xi_{i}}\right|} D\left(\left.v\right|_{\tau}\right),
$$


with the notation defined at the end of $\S 2.2$. For a function defined at the mesh nodes, we set

$$
\left[D_{h} v\right]\left(\xi_{i}\right)=\sum_{\tau \in \mathcal{J}_{h, i}} \frac{|\tau|}{\left|\omega_{\xi_{i}}\right|} D\left(\left.\ell_{h}[v]\right|_{\tau}\right)
$$

where $\ell_{h}$ is the Lagrange interpolation operator on piecewise affine functions. Note that $\left[D_{h} v\right]\left(\xi_{i}\right)$ can also be written

$$
\left|\omega_{\xi_{i}}\right|\left[D_{h} v\right]\left(\xi_{i}\right)=\left(\begin{array}{l}
\int_{\omega_{\xi_{i}}} D d_{h}[v] \cdot e_{1} \\
\int_{\omega_{\xi_{i}}} D \ell_{h}[v] \cdot e_{2}
\end{array}\right)=\left(\begin{array}{l}
\sum_{j \neq i} v\left(\xi_{j}\right) \int_{\omega_{\xi_{i}}} D \lambda_{j} \cdot e_{1} \\
\sum_{j \neq i} v\left(\xi_{j}\right) \int_{\omega_{\xi_{i}}} D \lambda_{j} \cdot e_{2}
\end{array}\right) .
$$

It can be proved that this is a first order approximation, i.e. $q=1$ in (15). Moreover, it is well known that if the mesh is uniform and made with right isocele triangles, then the approximation is superconvergent, i.e., $q=2$.

REMARK 2 In general, it is possible to construct second order approximations of the gradient of $v$ at $\xi_{i}$ by linear combinations of the values $v\left(\xi_{j}\right)$, where $\xi_{j}$ are the vertices of $\omega_{\xi_{i}}$. The idea is to use a third order interpolation operator of $v$ on $\omega_{\xi_{i}}$ (exact on second order polynomials). Such an interpolation is not unique in general.

For each $i=1, \ldots, N_{h}$, set

$$
D_{i}^{n}=\left[D_{h} u^{n}\right]\left(\xi_{i}\right)
$$

Given two positive numbers $C$ and $s$, the two sets of indices $g_{1}^{n}$ and $g_{2}^{n}$ are defined as follows:

$$
\begin{aligned}
& g_{1}^{n}=\left\{i=1, \ldots, N_{h} ; \xi_{i} \text { is strongly internal and }\left|D_{i}^{n}\right| \geqslant C h^{s}\right\}, \\
& g_{2}^{n}=\left\{i=1, \ldots, N_{h} ; \xi_{i} \text { is strongly internal and }\left|D_{i}^{n}\right|<C h^{s}\right\} .
\end{aligned}
$$

If $i \in g_{1}^{n}$, then we introduce the unit vector $\Theta_{i}^{n}$ by

$$
\Theta_{i}^{n}=\frac{1}{\left|D_{i}^{n}\right|}\left(\begin{array}{r}
-\left(D_{i}^{n}\right)_{2} \\
\left(D_{i}^{n}\right)_{1}
\end{array}\right)
$$

and we compute $u_{i}^{n+1}$ by

$$
u_{i}^{n+1}=u_{i}^{n}+\frac{\Delta t}{\delta^{2}}\left(\ell_{h}\left[u^{n}\right]\left(\xi_{i}+\delta \Theta_{i}^{n}\right)+\ell_{h}\left[u^{n}\right]\left(\xi_{i}-\delta \Theta_{i}^{n}\right)-2 u_{i}^{n}\right), \quad i \in \mathcal{I}_{1}^{n},
$$

which is possible thanks to (9).

REMARK 3 It is useful to recall that the mapping $\Theta: p \mapsto \frac{1}{|p|}\left(p_{2},-p_{1}\right)^{T}$ is differentiable in $\mathbb{R}^{2} \backslash\{0\}$ and that its Jacobian matrix at $p \neq 0$ is

$$
D \Theta(p)=\left(\begin{array}{cc}
-\frac{p_{1} p_{2}}{\mid p} & \frac{p_{1}^{2}}{\mid p p^{3}} \\
-\frac{p_{2}^{2}}{|p|^{3}} & \frac{p_{1} p_{2}}{|p|^{3}}
\end{array}\right),
$$

so the Frobenius norm of $D \Theta(p)$ equals $1 /|p|$. 
REMARK 4 Concerning the implementation of (23), the main difficulty is to locate the triangle containing the feet of the (generalized) characteristics, namely $\xi_{i} \pm \delta \Theta_{i}^{n}$ in order to compute the value of the solution at that point via a local interpolation operator. Trying to locate $z \equiv \xi_{i}+\delta \Theta_{i}^{n}$ for example, one can start from a triangle containing $\xi_{i}$ and construct inductively a sequence $\left(\tau_{m}\right)$ of triangles closer and closer to $z$ as follows: if $\tau_{m}$ contains $z$, then stop. Else, call $\left(A_{j}\right)_{j=1,2,3}$ the vertices of $\tau_{m}$ and $\left(w_{j}\right)_{j=1,2,3}$ the related barycentric coordinates. Compute the numbers $w_{j}(z)$. If there exists only one index $j$ such that $w_{j}(z)<0$, say $j=1$, then $\tau_{m+1}$ is the triangle sharing the edge $A_{2} A_{3}$ with $\tau_{m}$. If on the contrary, we have $w_{j}(z)<0$ for two indices $j$, say $j=1$ and $j=2$, then choose $\tau_{m+1}$ as one of the two triangles sharing with $\tau_{m}$ the edges $A_{1} A_{3}$ or $A_{2} A_{3}$.

The values $u_{i}^{n+1}, i \in \mathcal{G}_{2}^{n}$ remain to be defined. We set

$$
u_{i}^{n+1}=-\frac{\sum_{j \neq i} A_{i j} u_{j}^{n}}{A_{i i}}=\frac{\sum_{j \neq i} A_{i j} u_{j}^{n}}{\sum_{j \neq i} A_{i j}}, \quad i \in \mathcal{I}_{2}^{n},
$$

where the matrix $A$ has been defined in (10). We can also write (24) in the following way:

$$
\left(\sum_{j=1}^{N_{h}} M_{i j}\right)\left(u_{i}^{n+1}-u_{i}^{n}\right)=-\epsilon_{i} \Delta t \sum_{j=1}^{N_{h}} A_{i j} u_{j}^{n},
$$

where

$$
\epsilon_{i}=\frac{\left(\sum_{j=1}^{N_{h}} M_{i j}\right)}{\Delta t A_{i i}}
$$

We see that $u_{i}^{n+1}$ is found by performing one iteration of an explicit Euler scheme for a parabolic equation of the form

$$
\frac{\partial w}{\partial t}-\epsilon(x) \Delta w=0,
$$

with mass lumping (we have replaced the mass matrix which is involved in the Galerkin approximation of (26) by a diagonal matrix whose $i^{\text {th }}$ diagonal coefficient is obtained by summing up all the coefficients of the $i^{t h}$ row of the mass matrix $M$ ), and where $\epsilon$ is a small (of the order of $h^{2} / \Delta t$ ) variable coefficient.

To summarize, if $\xi_{i}$ is a strongly internal node,

$$
\begin{array}{rlrl}
u_{i}^{n+1} & =u_{i}^{n}+\frac{\Delta t}{\delta^{2}}\left(\ell_{h}\left[u^{n}\right]\left(\xi_{i}+\delta \Theta_{i}^{n}\right)+\ell_{h}\left[u^{n}\right]\left(\xi_{i}-\delta \Theta_{i}^{n}\right)-2 u_{i}^{n}\right), & & \text { if } i \in \mathcal{Y}_{1}^{n}, \\
u_{i}^{n+1}=-A_{i i}^{-1} \sum_{j \neq i} A_{i j} u_{j}^{n}, & & \text { if } i \in \mathcal{Y}_{2}^{n} .
\end{array}
$$

For a given index $i=1, \ldots, N_{h}$, we write the nonlinear equation describing the scheme at node $\xi_{i}$ (i.e. (14) if $\xi_{i} \in \Gamma_{h}^{\ell}$ ), (27) if $\xi_{i}$ is a strongly internal node) in the generic form

$$
\mathrm{g}^{\Delta t}\left(i, n, u^{n+1}, u^{n}\right)=0, \quad i=1, \ldots, N_{h}
$$

REMARK 5 The scheme at strongly inner nodes can be interpreted in various ways: first, the method introduced in [18] is used as long as $|D u|$ is large enough: the stochastic dynamical system which is behind the degenerate parabolic operator is discretized and we obtain a system of "generalized 
characteristics" for the degenerate problem. The stochastic problem behind this interpretation has been introduced and analyzed by Buckdahn, Cardaliaguet and Quincampoix in [6] and Soner and Touzi in [29, 30]. More recently, Kohn and Serfaty have given in [22] a discrete game interpretation, in which the degenerate parabolic operator is approximated by a time discretization of min-max type.

Second, in order to handle the singular case $|D u|=0$, the scheme proposed in [18] is modified by switching to an approximation of the heat equation whenever $|D u|$ is below a given threshold.

2.3.3 Property. Invariance with respect to the addition of constants.

If we write the global scheme in the form

$$
u^{n+1}=\delta^{\Delta t}\left(u^{n}\right),
$$

then it is easy to prove that, for all real number $k$, we have,

$$
\delta^{\Delta t}\left(u^{n}+k\right)=\delta^{\Delta t}\left(u^{n}\right)+k .
$$

REMARK 6 It is interesting to note that, if Assumption 1 does not hold, then one may wish to use a scheme different from (24): it is well known that there exists a field of symmetric tensors $x \rightarrow a(x)$ such that $\left.a\right|_{t}$ is constant for all $t \in \mathcal{J}_{h}$, and that there exist three positive constants $\alpha \leqslant \underline{a}<\bar{a}$ independent of $h$ such that for all $\xi \in \mathbb{R}^{2}$,

- $a$ satisfies the uniform continuity and ellipticity properties:

$$
|a(x) \xi| \leqslant \bar{a}|\xi|, \quad \text { and } \quad \xi^{T} a(x) \xi \geqslant \underline{a}|\xi|^{2} ;
$$

- the matrix $\widetilde{A} \in \mathbb{R}^{N_{h} \times N_{h}}: \widetilde{A}_{i, j}=\int_{\Omega_{h}} a(x) D \lambda_{i}(x) \cdot D \lambda_{j}(x) d x$ is a M-matrix. Moreover, if $\xi_{i} \neq \xi_{j}$ are two vertices of a same triangle $t \in \mathcal{J}_{h}$, then $\widetilde{A}_{i, j} \leqslant-\frac{\alpha}{h^{2}}$.

Then, instead of (24), one may use

$$
u_{i}^{n+1}=-\frac{\sum_{j \neq i} \widetilde{A}_{i j} u_{j}^{n}}{\widetilde{A}_{i i}}=\frac{\sum_{j \neq i} \widetilde{A}_{i j} u_{j}^{n}}{\sum_{j \neq i} \widetilde{A}_{i j}}, \quad i \in \mathcal{G}_{2}^{n} .
$$

\subsection{An example of a Godunov like scheme for (12)}

Let $\xi_{i}$ belong to $\Xi_{h}^{\ell}$. For simplicity, when writing the scheme, we drop the index accounting for time. To recover a scheme of the form (14), all the values of $u$ related to boundary nodes should be taken at time $t_{n+1}$ and all the values related to strongly internal nodes should be taken at time $t_{n}$.

If $\xi_{i} \notin \Gamma^{\ell}$, the line $\xi_{i}+\mathbb{R} n^{\ell}\left(\xi_{i}\right)$ cuts the polygonal line $\partial \omega_{\xi_{i}}$ at two points $\xi_{i}^{\nu,+}$ and $\xi_{i}^{\nu,-}$, with $\left(\xi_{i}^{\nu,+}-\xi_{i}\right) \cdot n^{\ell}\left(\xi_{i}\right)>0$ and $\left(\xi_{i}^{\nu,-}-\xi_{i}\right) \cdot n^{\ell}\left(\xi_{i}\right)<0$. We use the notation $h_{i}^{\nu, \pm}=\left|\xi_{i}^{\nu, \pm}-\xi_{i}\right|$. Similarly, if $t^{\ell}\left(\xi_{i}\right)=\left(-n_{2}^{\ell}\left(\xi_{i}\right), n_{1}^{\ell}\left(\xi_{i}\right)\right)$, the line $\xi_{i}+\mathbb{R} t^{\ell}\left(\xi_{i}\right)$ cuts the polygonal line $\partial \omega \xi_{i}$ at two points $\xi_{i}^{\tau,+}$ and $\xi_{i}^{\tau,-}$, with $\left(\xi_{i}^{\tau,+}-\xi_{i}\right) \cdot t^{\ell}\left(\xi_{i}\right)>0$ and $\left(\xi_{i}^{\tau,-}-\xi_{i}\right) \cdot t^{\ell}\left(\xi_{i}\right)<0$. We use the notation $h_{i}^{\tau, \pm}=\left|\xi_{i}^{\tau, \pm}-\xi_{i}\right|$. An example is given in Figure 3 .

We use the following finite differences:

$$
\begin{aligned}
D_{i}^{\nu,+} u & =\frac{1}{h_{i}^{\nu,+}}\left(\ell_{h}[u]\left(\xi_{i}^{\nu,+}\right)-u_{i}\right), & D_{i}^{\nu,-} u & =\frac{1}{h_{i}^{\nu,-}}\left(u_{i}-\ell_{h}[u]\left(\xi_{i}^{\nu,-}\right)\right), \\
D_{i}^{\tau,+} u & =\frac{1}{h_{i}^{\tau,+}}\left(\ell_{h}[u]\left(\xi_{i}^{\tau,+}\right)-u_{i}\right), & D_{i}^{\tau,-} u & =\frac{1}{h_{i}^{\tau,-}}\left(u_{i}-\ell_{h}[u]\left(\xi_{i}^{\tau,-}\right)\right) .
\end{aligned}
$$




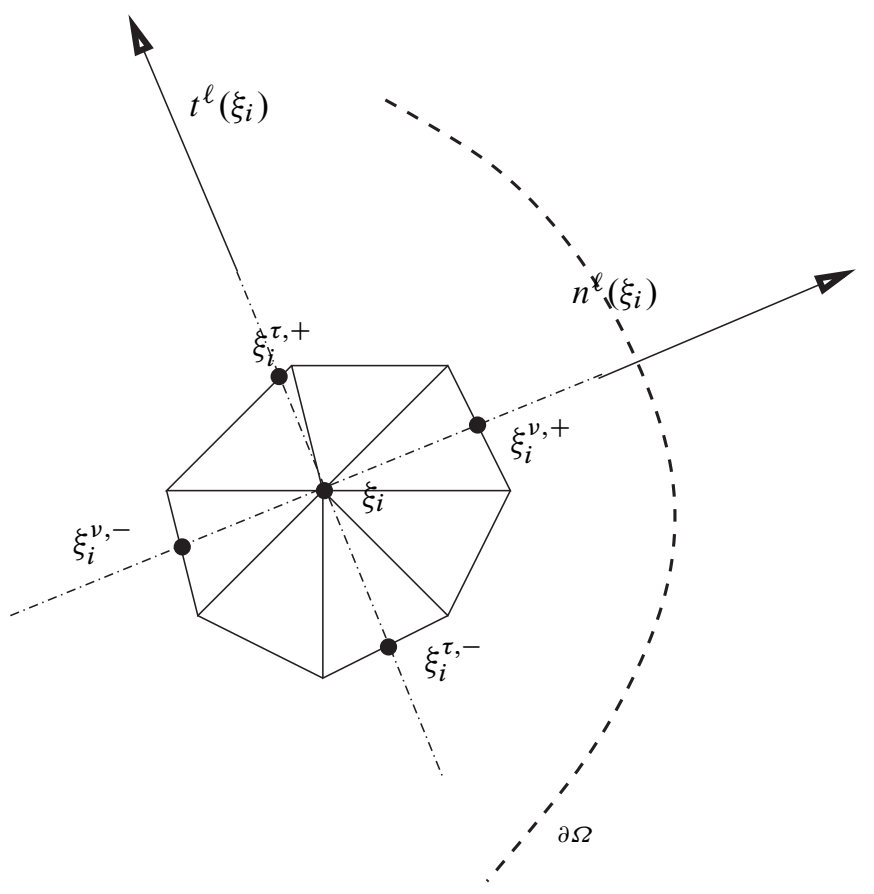

FIG. 3. The construction of the scheme at $\xi_{i} \in \Xi_{h}^{\ell} \backslash \Gamma^{\ell}$

If $\theta<0$, then the Godunov scheme at $\xi_{i}$ is:

$$
D_{i}^{\nu,-} u-\theta\left(\min \left(D_{i}^{v,+} u, 0\right)^{2}+\max \left(D_{i}^{\nu,-} u, 0\right)^{2}+\min \left(D_{i}^{\tau,+} u, 0\right)^{2}+\max \left(D_{i}^{\tau,-} u, 0\right)^{2}\right)^{\frac{1}{2}}=0 .
$$

If $\theta>0$, then the Godunov scheme at $\xi_{i}$ is:

$$
D_{i}^{v,-} u-\theta\left(\max \left(D_{i}^{v,+} u, 0\right)^{2}+\min \left(D_{i}^{v,-} u, 0\right)^{2}+\max \left(D_{i}^{\tau,+} u, 0\right)^{2}+\min \left(D_{i}^{\tau,-} u, 0\right)^{2}\right)^{\frac{1}{2}}=0
$$

If on the contrary, $\xi_{i}$ lies on $\Gamma_{\ell}$, then we define $\xi_{i}^{\nu,-}$ and $h_{i}^{\nu,-}$ as above, but the nodes $\xi_{i}^{\tau, \pm}$ are now the two neighbors of $\xi_{i}$ on $\Gamma_{\ell}$; we take $h_{i}^{\tau, \pm}=\left|\xi_{i}^{\tau, \pm}-\xi_{i}\right|$. If $\theta<0$, then the upwind scheme at $\xi_{i}$ is:

$$
D_{i}^{v,-} u-\theta\left(\min \left(D_{i}^{\tau,+} u, 0\right)^{2}+\max \left(D_{i}^{\tau,-} u, 0\right)^{2}+\left(D_{i}^{v,-} u\right)^{2}\right)^{\frac{1}{2}}=0 .
$$

If $\theta>0$, then the Godunov scheme at $\xi_{i}$ is:

$$
D_{i}^{v,-} u-\theta\left(\max \left(D_{i}^{\tau,+} u, 0\right)^{2}+\min \left(D_{i}^{\tau,-} u, 0\right)^{2}+\left(D_{i}^{v,-} u\right)^{2}\right)^{\frac{1}{2}}=0
$$

\section{Analysis of the scheme}

Our goal is to establish the convergence of an approximation scheme obtained by adding an articial viscosity regularization to (27)-(28), via the abstract result proved in [7] (see Section 2 there for 
more details). The modified scheme will be defined in $\S 3.2$ below, see (47). We will prove that this scheme is consistent and monotone.

Let us recall, for readers convenience, the definition of viscosity solution for the boundary value problem. We denote by $\underline{F}(p, X)$ and $\bar{F}(p, X)$ the lower and upper semicontinuous extensions of the function $F(p, X)=-\operatorname{trace}\left(\left(I-\frac{p \otimes p}{|p|^{2}}\right) X\right)$ at $p=0$. With the notation:

$$
\begin{aligned}
& \bar{G}(x, \eta, p, X)=\left\{\begin{array}{l}
\eta+\bar{F}(p, X) \text { if } x \in \Omega, \\
\max (\eta+\bar{F}(p, X), p \cdot n-\theta|p|)
\end{array} \text { if } x \in \partial \Omega,\right. \\
& \underline{G}(x, \eta, p, X)=\left\{\begin{array}{l}
\eta+\underline{F}(p, X) \text { if } x \in \Omega, \\
\min (\eta+\underline{F}(p, X), p \cdot n-\theta|p|)
\end{array} \text { if } x \in \partial \Omega,\right.
\end{aligned}
$$

and following [11], we say that an upper semicontinuous function $u$ is a subsolution if for all $\Phi \in$ $\mathrm{e}^{1}(\bar{\Omega} \times(0, T])$, if $\left(x_{0}, t_{0}\right)$ is a maximum point of $u-\Phi$ then

$$
\underline{G}\left(x_{0}, \frac{\partial \Phi}{\partial t}\left(x_{0}, t_{0}\right), D \Phi\left(x_{0}, t_{0}\right), D^{2} \Phi\left(x_{0}, t_{0}\right)\right) \leqslant 0 ;
$$

we say that a lower semicontinuous function $u$ is a supersolution if for all $\Phi \in \mathrm{C}^{1}(\bar{\Omega} \times(0, T])$, if $\left(x_{0}, t_{0}\right)$ is a minimum point of $u-\Phi$ then

$$
\bar{G}\left(x_{0}, \frac{\partial \Phi}{\partial t}\left(x_{0}, t_{0}\right), D \Phi\left(x_{0}, t_{0}\right), D^{2} \Phi\left(x_{0}, t_{0}\right)\right) \geqslant 0 .
$$

\subsection{Consistency}

We are going to prove the consistency of the scheme (28). The consistency of the modified scheme introduced in $\S 3.2$ below is obtained in the same way, since it only differs from the present one by the addition of a vanishing artificial viscosity. Following Barles and Souganidis [5], we say that the scheme (28) is consistent if for any smooth function $\Phi$ defined on $[0, T] \times \bar{\Omega}$, we have that for any $t \in(0, T]$ and $x \in \bar{\Omega}$, for any sequence of positive mesh parameters $\left(h_{m}, \Delta t_{m}, \delta_{m}\right)$ tending to 0 with $h_{m}=o\left(\delta_{m}\right)$, for any sequence $\xi_{i, m}$ tending to $x$ as $m \rightarrow \infty, \xi_{i, m}$ being a mesh node of $\mathcal{J}_{h_{m}}$, and for any sequence $t_{n_{m}}=n_{m} h_{m}$ tending to $t$ as $m$ tends to $\infty$,

$$
\begin{aligned}
\underline{G}\left(x, \frac{\partial \Phi}{\partial t}(x, t), D \Phi(x, t), D^{2} \Phi(x, t)\right) & \leqslant \liminf _{m \rightarrow \infty} \mathcal{G}^{\Delta t}\left(i_{m}, n_{m}, \Phi^{n_{m}+1}, \Phi^{n_{m}}\right) \\
& \leqslant \limsup _{m \rightarrow \infty} \mathcal{G}^{\Delta t}\left(i_{m}, n_{m}, \Phi^{n_{m}+1}, \Phi^{n_{m}}\right) \\
& \leqslant \bar{G}\left(x, \frac{\partial \Phi}{\partial t}(x, t), D \Phi(x, t), D^{2} \Phi(x, t)\right),
\end{aligned}
$$

calling $\Phi^{n}=\left(\Phi\left(\xi_{j}, n \Delta t\right)\right)_{j=1, \ldots, N_{h}}$.

Proposition 1 Assume that $h^{2} / \Delta t=o(1), h / \delta=o(1)$ and $h^{q-s} / \delta=o(1)$. Then the scheme is consistent.

Proof. Consider a sequence of positive mesh parameters $\left(h_{m}, \Delta t_{m}, \delta_{m}\right)$ tending to 0 with $h_{m}=$ $o\left(\delta_{m}\right)$, a sequence $\xi_{i, m}$ (tending to $x$ as $m \rightarrow \infty, \xi_{i, m}$ being a mesh node of $\tau_{h_{m}}$, and a sequence $t_{n_{m}}=n_{m} h_{m}$ tending to $t$ as $h$ tends to 0 . For brevity, we will drop the index $m$ when there is no ambiguity.

We can make out four cases: 
Case 1: $x \in \Omega$ and $D \Phi(x, t) \neq 0 \quad$ In this case, we know that there exists $\rho>0$ and $\kappa>0$ such that the ball $B(x, \rho)$ is contained in $\Omega$ and that $|D \Phi(y, s)|>\kappa$ for all $y \in B(x, \rho)$ and $s \in[\min (0, t-$ $\rho), \max (T, t+\rho)]$. Since $\left[D_{h} \Phi(\cdot, t)\right]$ is a consistent approximation of the gradient $D \Phi(\cdot, t)$, we know that if $h$ and $\Delta t$ are small enough, then for all the mesh nodes $\xi$ contained in e.g. $B(x, \rho / 2)$, and for all the discrete times $t_{n} \in[\min (0, t-\rho / 2), \max (T, t+\rho / 2)]$, we have $\left|\left[D_{h} \Phi\left(\cdot, t_{n}\right)\right](\xi)\right|>$ $C h^{s}$, where $C$ and $s$ are the constants used in (21). Moreover, we can choose $\delta$ small enough such that all the mesh nodes contained in $B(x, \rho / 2)$ are internal mesh nodes. Therefore, for $m$ large enough, for all the mesh nodes $\xi_{i}$ contained in the ball $B(x, \rho / 2)$ and for all the discrete times $t_{n} \in[\min (0, t-\rho / 2), \max (T, t+\rho / 2)]$,

$$
\begin{aligned}
\mathcal{G}^{\Delta t}\left(i, n, \Phi^{n+1}, \Phi^{n}\right) & \\
\quad= & \frac{\Phi\left(\xi_{i}, t_{n+1}\right)-\Phi\left(\xi_{i}, t_{n}\right)}{\Delta t}+\frac{2 \Phi\left(\xi_{i}, t_{n}\right)-\ell_{h}\left[\Phi^{n}\right]\left(\xi_{i}+\delta \Theta_{i}^{n}\right)-\ell_{h}\left[\Phi^{n}\right]\left(\xi_{i}-\delta \Theta_{i}^{n}\right)}{\delta^{2}}
\end{aligned}
$$

where $\Theta_{i}^{n}$ is given by (22) with $D_{i}^{n}=\left[D_{h} \Phi^{n}\right]\left(\xi_{i}\right)$.

Then, using the results contained in Carlini et al [7], we have

$$
\lim _{m \rightarrow \infty} g^{\Delta t}\left(i, n, \Phi^{n+1}, \Phi^{n}\right)=\left(\frac{\partial \Phi}{\partial t}-\operatorname{trace}\left(\left(I-\frac{D \Phi \otimes D \Phi}{|D \Phi|^{2}}\right) D^{2} \Phi\right)\right)(x, t),
$$

which yields (32). In fact, following [7], it can be seen that, if $D \Phi(x, t) \neq 0$, then for $m$ large enough and $\left|\xi_{i}-x\right| \sim h,\left|t-t_{n}\right| \sim \Delta t$,

$$
\begin{aligned}
\mid g^{\Delta t}\left(i, n, \Phi^{n+1}, \Phi^{n}\right)-\left(\frac{\partial \Phi}{\partial t}-\operatorname{trace}((I-\right. & \left.\left.\left.\frac{D \Phi \otimes D \Phi}{|D \Phi|^{2}}\right) D^{2} \Phi\right)\right)(x, t) \mid \\
& =O\left(\frac{h^{2}}{\delta^{2}}\right)+O\left(\frac{h^{q}}{\delta}\right)+O(\Delta t)+O\left(\delta^{2}\right)
\end{aligned}
$$

Case 2: $x \in \Omega$ and $D \Phi(x, t)=0$ a) Let us first suppose that for all $m$ large enough, $D_{i}^{n}=$ $\left[D_{h} \Phi^{n}\right]\left(\xi_{i}\right)$ is such that $\left|D_{i}^{n}\right| \geqslant C h^{s}$. If $s<q$, then for $h$ small enough, $D \Phi\left(\xi_{i}, t_{n}\right) \neq 0$, because the discrete gradient is an approximation of order $q$ of the gradient. In this case, we have (33), and following [7], this implies that

$$
\begin{aligned}
\mid g^{\Delta t}\left(i, n, \Phi^{n+1}, \Phi^{n}\right)-\left(\frac{\partial \Phi}{\partial t}-\operatorname{trace}((I\right. & \left.\left.\left.-\frac{D \Phi \otimes D \Phi}{|D \Phi|^{2}}\right) D^{2} \Phi\right)\right)\left(\xi_{i}, t_{n}\right) \mid \\
& =O\left(\frac{h^{2}}{\delta^{2}}\right)+O\left(\frac{h^{q-s}}{\delta}\right)+O(\Delta t)+O\left(\delta^{2}\right) .
\end{aligned}
$$

Since $h^{q-s}=o(\delta)$, this implies that

$$
\begin{aligned}
\frac{\partial \Phi}{\partial t}(x, t)+\underline{F}\left(0, D^{2} \Phi(x, t)\right) \leqslant & \liminf _{m \rightarrow \infty} g^{\Delta t}\left(i, n, \Phi^{n+1}, \Phi^{n}\right) \\
\leqslant & \limsup _{m \rightarrow \infty} g^{\Delta t}\left(i, n, \Phi^{n+1}, \Phi^{n}\right) \\
& \leqslant \frac{\partial \Phi}{\partial t}(x, t)+\bar{F}\left(0, D^{2} \Phi(x, t)\right) .
\end{aligned}
$$


b) Let us now suppose that for all $m$ large enough, $D_{i}^{n}=\left[D_{h} \Phi^{n}\right]\left(\xi_{i}\right)$ is such that $\left|D_{i}^{n}\right|<C h^{s}$. Thus,

$$
g^{\Delta t}\left(i, n, \Phi^{n+1}, \Phi^{n}\right)=\frac{1}{\Delta t}\left(\Phi_{i}^{n+1}-\Phi_{i}^{n}\right)+\epsilon_{i}\left(\sum_{j=1}^{N_{h}} M_{i j}\right)^{-1} \sum_{j=1}^{N_{h}} A_{i j} \Phi_{j}^{n} .
$$

where

$$
\epsilon_{i}=\frac{\left(\sum_{j=1}^{N_{h}} M_{i j}\right)}{\Delta t A_{i i}}
$$

It is simple to prove that if $h^{2} / \Delta t=o(1)$ then

$$
\lim _{m \rightarrow \infty} \mathrm{g}^{\Delta t}\left(i, n, \Phi^{n+1}, \Phi^{n}\right)=\frac{\partial \Phi}{\partial t}(x, t)
$$

which implies (37).

From points a) and b), we easily deduce (32) when $D \Phi(x, t)=0$.

Case 3: $x \in \Gamma^{\ell} \subset \partial \Omega$ and $D \Phi(x, t) \neq 0 \quad$ In this case, we know that there exists $\rho>0$ and $\kappa>0$ such that $|D \Phi(y, s)|>\kappa$ for all $y \in B(x, \rho) \cap \bar{\Omega}$ and $s \in[\min (0, t-\rho)$, $\max (T, t+\rho)]$.

a) Suppose that $m$ large enough, $\xi_{i}$ is a strictly internal mesh node: in this case, we can replicate the arguments used in Case 1: for $m$ large enough we may assume that $\xi_{i} \in B(\xi, h / 2) \cap \Omega$ and obtain that

$$
\lim _{m \rightarrow \infty} g^{\Delta t}\left(i, n, \Phi^{n+1}, \Phi^{n}\right)=\left(\frac{\partial \Phi}{\partial t}-\operatorname{trace}\left(\left(I-\frac{D \Phi \otimes D \Phi}{|D \Phi|^{2}}\right) D^{2} \Phi\right)\right)(x, t) .
$$

b) Suppose that for $m$ large enough, $\xi_{i}$ belongs to $\Xi_{h}^{\ell}$, so

$$
\mathrm{G}^{\Delta t}\left(i, n, \Phi^{n+1}, \Phi^{n}\right)=\mathbb{B}^{\ell}\left(\xi_{y}, \Phi\left(\xi_{i}, t_{n+1}\right),\left[\Phi^{n+1}\right] \ell,\left[\left[\Phi^{n}\right]\right]\right)
$$

see (13) for the notations. Since this scheme is consistent with (12), we see that

$$
\lim _{m \rightarrow \infty} \mathrm{g}^{\Delta t}\left(i, n, \Phi^{n+1}, \Phi^{n}\right)=\frac{\partial \Phi}{\partial n^{\ell}}(x, t)-\theta|D \Phi(x, t)| .
$$

From the results obtained in points a) and b), it is an easy matter to deduce (32) by extracting subsequences if necessary.

Case 4: $x \in \Gamma^{\ell} \subset \partial \Omega$ and $D \Phi(x, t)=0$ We argue as in Case 3, but we now have to consider three kinds of sequences $\left(\xi_{i}, t_{n}\right)$ :

a) Suppose that for $m$ large enough, $\xi_{i}$ is a strictly internal mesh node, and $D_{i}^{n}=\left[D_{h} \Phi^{n}\right]\left(\xi_{i}\right)$ is such that $\left|D_{i}^{n}\right| \geqslant C h^{s}$. In this case, we argue as in Case 2:a).

b) Suppose that for $m$ large enough, $\xi_{i}$ is a strictly internal mesh node, and $D_{i}^{n}=\left[D_{h} \Phi^{n}\right]\left(\xi_{i}\right)$ is such that $\left|D_{i}^{n}\right|<C h^{s}$. In this case, we argue as in Case 2:b).

c) Suppose that for $m$ large enough, $\xi_{i}$ belongs to $\Xi_{h}^{\ell}$. We argue as in Case 3:b).

From the points a), b) and c), we deduce (32). 


\subsection{Monotonicity}

To discuss the monotonicity, we suppose that Assumption 1 holds and we restrict ourselves to taking for $\left[D_{h} u\right]$ the first order approximation of the gradient defined in (19), so $q=1$. Hereafter, we suppose that

$$
h^{1-s}=o(\delta) \text {. }
$$

The standard definition of monotonicity is also replaced by a generalized monotonicity assumption stated as follows.

DEFInITION 3 The scheme $S^{\Delta t}$ is said to be monotone (in the generalized sense) if it satisfies the following conditions: let $\left(h_{m}, \Delta t_{m}, \delta_{m}\right)$ and $\left(\xi_{j_{m}}, t_{n_{m}}\right)$ be generic sequences satisfying

$$
\left(h_{m}, \Delta t_{m}, \delta_{m}\right) \rightarrow 0 \quad \text { and } \quad\left(\xi_{j_{m}}, t_{n_{m}}\right) \rightarrow(\xi, t) .
$$

Then, for any smooth function $\phi$,

$$
\begin{aligned}
& \text { if } v_{j_{m}} \leqslant \phi_{j_{m}}^{n_{m}-1} \text { then } S^{\Delta t_{m}}\left(v ; j_{m}\right) \leqslant \widetilde{S}^{\Delta t_{m}}\left(\phi^{n_{m}-1} ; j_{m}\right)+o\left(\Delta t_{m}\right) \\
& \text { if } \phi_{j}^{n_{m}-1} \leqslant v_{j_{m}} \text { then } \widetilde{S}^{\Delta t_{m}}\left(\phi^{n_{m}-1} ; j_{m}\right) \leqslant S^{\Delta t_{m}}\left(v ; j_{m}\right)+o\left(\Delta t_{m}\right),
\end{aligned}
$$

where $v$ is a set of node values, and $\widetilde{S}^{\Delta t}$ is a (possibly different) scheme consistent in the sense that it satisfies $(32)$ in $(\xi, t)$.

In the sequel, we write the scheme (14), (27) in the following compact form:

$$
u_{i}^{n+1}=\delta\left(u^{n} ; i\right) .
$$

Following the ideas contained in [12], we will consider a scheme which results from the further introduction of a vanishing artificial viscosity in (46). The motivation for such an adaptation is the following. The scheme (23) is monotone only with respect to the nodes involved by the interpolation operator. On the other hand, the dependence on the values involved in the approximation of the gradient is more complex, and the introduction of the artificial viscosity term allows to gain monotonicity with respect to these values.

Therefore, we will prove the generalized monotonicity property for the modified scheme $u_{i}^{n+1}=\widehat{\delta}\left(u^{n} ; i\right)$ : the scheme is not modified at boundary nodes, and if $\xi_{i}$ is a strongly internal node, then

$$
\begin{aligned}
\widehat{\delta}\left(u^{n} ; i\right) & =\delta\left(u^{n} ; i\right)-W \frac{\Delta t}{\delta h^{s+1}} \sum_{j=1}^{N_{h}} A_{i j} u_{j}^{n}, & & \text { if } i \in \mathcal{I}_{1}^{n}, \\
& =-\left(A_{i i}\right)^{-1} \sum_{j \neq i} A_{i j} u_{j}^{n}, & & \text { if } i \in \mathcal{Y}_{2}^{n},
\end{aligned}
$$

where $W$ is a suitable positive constant.

REMARK 7 Note that $\frac{\Delta t}{\delta h^{s+1}} \sum_{j=1}^{N_{h}} A_{i j} u_{j}^{n}$ is actually a vanishing viscosity term if $h^{1-s}=o(\delta)$ : indeed, remember that in $\frac{1}{h^{2}} \sum_{j \neq i} A_{i j} u_{j}^{n}$ can be viewed as a discrete version of the partial differential operator $-\Delta$ applied to the grid function $u^{n}$ at $\xi_{i}$. Therefore $\frac{\Delta t}{\delta h^{s+1}} \sum_{j=1}^{N_{h}} A_{i j} u_{j}^{n}$ plays the role of $-\Delta t \frac{h^{1-s}}{\delta} \Delta u\left(\xi_{i}, t_{n}\right)$. The artificial viscosity is thus of the order of $h^{1-s} \delta^{-1}$. 
We do not need to consider boundary nodes, because the scheme (13) is monotone (in the classical sense). We thus focus on strongly internal nodes.

First inequality To check (44), suppose now that for any sequence $\left(\xi_{j}, t_{n}\right) \equiv\left(\xi_{j_{m}}, t_{n_{m}}\right)$ verifying (43), the following inequality holds

$$
u_{j}^{n} \leqslant \varphi_{j}^{n} \equiv \Phi\left(\xi_{j}, t_{n}\right)
$$

where $u^{n}$ is the solution of (14) and (27), for test functions $\Phi \in C^{\infty}(\bar{\Omega} \times[0, T])$. Since the monotonicity property does not depend on the iteration $n$, then, with no loss of generality, we can drop the dependency on $t$ of the test function $\Phi$. Condition (44) can be recast in the form

$$
\widehat{\delta}\left(u^{n} ; i\right) \leqslant \widetilde{\delta}(\varphi ; i)+o(\Delta t),
$$

where the choice of $\widetilde{S}$ may vary from one subcase to the other. We will often omit the time index, writing $u$ for $u^{n}$.

The proof discriminates between two main cases.

Case $1 \quad D \Phi(\xi) \neq 0$.

a) $\xi_{i}$ is a strongly internal node and $\left|\left[D_{h} u\right]\left(\xi_{i}\right)\right| \geqslant C h^{s}$. Recall that we have denoted by $\Sigma_{i}$ the sets of node indices involved in the construction in $\left[D_{h} u\right]\left(\xi_{i}\right)$. It is clear that if $j \notin \Sigma_{i}$, then $\frac{\partial \delta(u ; i)}{\partial u_{j}} \geqslant 0$, from the monotonicity of the piecewise linear Lagrange interpolation operator $l_{h}$.

On the other hand, if $j \in \Sigma_{i}, j \neq i$, then calling $z_{i}^{ \pm}=\xi_{i} \pm \delta \Theta_{i}$, and assuming that $z_{i}^{ \pm}$do not lie on the boundary of a triangle of $\mathcal{J}_{h}$,

$$
\frac{\partial S(u ; i)}{\partial u_{j}}=\frac{\Delta t}{\delta}\left(D\left(\ell_{h}[u]\right)\left(z_{i}^{+}\right)-D\left(\ell_{h}[u]\right)\left(z_{i}^{-}\right)\right) \frac{\partial \Theta_{i}}{\partial u_{j}} .
$$

It can be proved that there exists a constant $L$ independent of $h$ such that if $\left|\left[D_{h} u\right]\left(\xi_{i}\right)\right| \geqslant C h^{s}$ and $j \in \Sigma_{i}$

$$
-L \frac{\Delta t}{\delta h^{s+1}} \leqslant \frac{\partial S(u ; i)}{\partial u_{j}} \leqslant L \frac{\Delta t}{\delta h^{s+1}} .
$$

Therefore, differentiating $\widehat{\delta}(u ; i)$, we get

$$
\frac{\partial \widehat{S}(u ; i)}{\partial u_{j}} \geqslant \frac{\Delta t}{\delta h^{s+1}}\left(-L-W A_{i j}\right) \geqslant \frac{\Delta t}{\delta h^{s+1}}(-L+W \alpha),
$$

where $\alpha$ is the constant appearing in (11). Similarly, for a positive constant $\bar{a}$,

$$
\frac{\partial \widehat{S}(u ; i)}{\partial u_{i}} \geqslant 1+\frac{2 \Delta t}{\delta^{2}}-\frac{\Delta t}{\delta h^{s+1}}\left(L+W A_{i i}\right) \geqslant 1+\frac{2 \Delta t}{\delta^{2}}-\frac{\Delta t}{\delta h^{s+1}}(L+W \bar{a}) .
$$

Therefore, if the conditions

$$
\begin{aligned}
-L+W \alpha & \geqslant 0, \\
1+\frac{2 \Delta t}{\delta^{2}}-\frac{\Delta t}{\delta h^{s+1}}(L+W \bar{a}) & \geqslant 0
\end{aligned}
$$


are fulfilled, then $\frac{\partial \widehat{\delta}(u ; i)}{\partial u_{j}} \geqslant 0$, for all $i$ such that $\left|\left[D_{h} u\right]\left(\xi_{i}\right)\right| \geqslant C h^{s}$ and for all $j$.

Then, we conclude as in [7].

b) $\left|\left[D_{h} u\right]\left(\xi_{i}\right)\right| \leqslant C h^{s}$. Since $\Phi \in C^{\infty}\left(\mathbb{R}^{2}\right)$, by a Taylor expansion we can write:

$$
\Phi(\xi)=\Phi\left(\xi_{i}\right)+\left(\xi-\xi_{i}\right) \cdot D \Phi\left(\xi_{i}\right)+\frac{1}{2}\left(\xi-\xi_{i}\right) \cdot D^{2} \Phi\left(\xi_{i}\right)\left(\xi-\xi_{i}\right)+O\left(\left|\xi-\xi_{i}\right|^{3}\right) .
$$

Therefore

$$
u_{j} \leqslant \Phi\left(\xi_{i}\right)+\left(\xi_{j}-\xi_{i}\right) \cdot D \Phi\left(\xi_{i}\right)+\frac{1}{2}\left(\xi_{j}-\xi_{i}\right) \cdot D^{2} \Phi\left(\xi_{i}\right)\left(\xi_{j}-\xi_{i}\right)+O\left(\left|\xi_{j}-\xi_{i}\right|^{3}\right) .
$$

On the other hand, we are going to use the special construction of $\left[D_{h} u\right]\left(\xi_{i}\right)$ given in (19):

$$
\left|\omega_{\xi_{i}}\right|\left[D_{h} u\right]\left(\xi_{i}\right)=\left(\begin{array}{l}
\sum_{j \neq i} u_{j} \int_{\omega_{\xi_{i}}} D \lambda_{j} \cdot e_{1} \\
\sum_{j \neq i} u_{j} \int_{\omega_{\xi_{i}}} D \lambda_{j} \cdot e_{2}
\end{array}\right) .
$$

Therefore, $\left|\left[D_{h} u\right]\left(\xi_{i}\right)\right| \leqslant C h^{s}$ implies that for any constant vector $\zeta$,

$$
\left|\sum_{j \neq i} u_{j} \int_{\omega_{\xi_{i}}} D \lambda_{j} \cdot \frac{\zeta}{h}\right| \leqslant C h^{s+1} .
$$

Hence,

$$
\begin{aligned}
-\sum_{j \neq i} A_{i, j} u_{j} & =-\int_{\omega_{\xi_{i}}} D d_{h}[u] \cdot D \lambda_{i}+u_{i} D \lambda_{i} \cdot D \lambda_{i} \\
& =-\sum_{j \neq i} u_{j} \int_{\omega_{\xi_{i}}} D \lambda_{j} \cdot D \lambda_{i} \\
& \leqslant-\sum_{j \neq i} u_{j} \int_{\omega_{\xi_{i}}} D \lambda_{j} \cdot\left(D \lambda_{i}-\frac{\zeta}{h}\right)+C h^{s+1}
\end{aligned}
$$

Under the assumptions on the mesh, it is always possible to choose $\zeta$ small enough such that

$$
C>-\int_{\omega_{\xi_{i}}} D \lambda_{j} \cdot\left(D \lambda_{i}-\frac{\zeta}{h}\right)>c>0, \quad \text { for all } i, j \in \Sigma_{i}, j \neq i
$$

With such a choice, exploiting the relations between the matrix involved in the artificial viscosity term and the linear interpolation operator of the gradient, we get

$$
\begin{aligned}
-\sum_{j \neq i} A_{i, j} u_{j} & \leqslant-\sum_{j \neq i} \Phi\left(\xi_{j}\right) \int_{\omega_{\xi_{i}}} D \lambda_{j} \cdot\left(D \lambda_{i}-\frac{\zeta}{h}\right)+C h^{s+1} \\
& =-\sum_{j \neq i} K_{j} \int_{\omega_{\xi_{i}}} D \lambda_{j} \cdot\left(D \lambda_{i}-\frac{\zeta}{h}\right)+C h^{s+1},
\end{aligned}
$$


where

$$
K_{j}=\Phi\left(\xi_{i}\right)+\left(\xi_{j}-\xi_{i}\right) \cdot D \Phi\left(\xi_{i}\right)+\frac{1}{2}\left(\xi_{j}-\xi_{i}\right) \cdot D^{2} \Phi\left(\xi_{i}\right)\left(\xi_{j}-\xi_{i}\right)+O\left(\left|\xi_{j}-\xi_{i}\right|^{3}\right)
$$

From (58), we deduce that

$$
\begin{aligned}
-\sum_{j \neq i} A_{i, j} u_{j} \leqslant & -\left(\sum_{j \neq i} A_{i, j}\right) \Phi\left(\xi_{i}\right)+\sum_{j \neq i}\left(\xi_{j}-\xi_{i}\right) \cdot D \Phi\left(\xi_{i}\right) \int_{\omega_{\xi_{i}}} D \lambda_{j} \cdot \frac{\zeta}{h} \\
& -\frac{1}{2} \sum_{j \neq i}\left(\xi_{j}-\xi_{i}\right) \cdot D^{2} \Phi\left(\xi_{i}\right)\left(\xi_{j}-\xi_{i}\right) \int_{\omega_{\xi_{i}}} D \lambda_{j} \cdot\left(D \lambda_{i}-\frac{\zeta}{h}\right)+O\left(h^{s+1}\right)
\end{aligned}
$$

because

$$
-\Phi\left(\xi_{i}\right) \sum_{j \neq i} \int_{\omega_{\xi_{i}}} D \lambda_{j} \cdot \frac{\zeta}{h}=\Phi\left(\xi_{i}\right) \int_{\omega_{\xi_{i}}} D \lambda_{i} \cdot \frac{\zeta}{h}=0
$$

and

$$
-\sum_{j \neq i}\left(\xi_{j}-\xi_{i}\right) \cdot D \Phi\left(\xi_{i}\right) \int_{\omega_{\xi_{i}}} D \lambda_{j} \cdot D \lambda_{i}=D \Phi\left(\xi_{i}\right) \cdot \int_{\omega_{\xi_{i}}} D \lambda_{i}=0 .
$$

Note also that

$$
\sum_{j \neq i}\left(\xi_{j}-\xi_{i}\right) \int_{\omega_{\xi_{i}}} D \lambda_{j} \cdot \frac{\zeta}{h}=\left|\omega_{\xi_{i}}\right| \frac{\zeta}{h}
$$

Hence, choosing now $\zeta=-\mu \frac{D \Phi\left(\xi_{i}\right)}{\left|D \Phi\left(\xi_{i}\right)\right|}$ with $\mu$ small enough such that (57) holds true yields

$$
\begin{aligned}
-\sum_{j \neq i} A_{i, j} u_{j}^{n} \leqslant & -\left(\sum_{j \neq i} A_{i, j}\right) \Phi\left(\xi_{i}\right)-\mu\left|D \Phi\left(\xi_{i}\right)\right| \frac{\left|\omega_{\xi_{i}}\right|}{h} \\
& -\frac{1}{2} \sum_{j \neq i}\left(\xi_{j}-\xi_{i}\right) \cdot D^{2} \Phi\left(\xi_{i}\right)\left(\xi_{j}-\xi_{i}\right) \int_{\omega_{\xi_{i}}} D \lambda_{j} \cdot\left(D \lambda_{i}-\frac{\zeta}{h}\right)+O\left(h^{s+1}\right) .
\end{aligned}
$$

Next, using the fact that $u^{n}$ satisfies (24), we add and subtract $\Delta t F\left(D \Phi(\xi), D^{2} \Phi(\xi)\right.$ ) (note that $\underline{F}=\bar{F}=F$, since we are in the case $D \Phi(\xi) \neq 0)$, obtaining

$$
\begin{aligned}
& u_{j}^{n+1} \leqslant \Phi\left(\xi_{i}\right)-\Delta t F\left(D \Phi(\xi), D^{2} \Phi(\xi)\right) \\
&-\frac{1}{\sum_{j \neq i} A_{i, j}}\left(\begin{array}{l}
-\mu\left|D \Phi\left(\xi_{i}\right)\right| \frac{\left|\omega_{\xi_{i}}\right|}{h}-\frac{1}{2} \sum_{j \neq i}\left(\xi_{j}-\xi_{i}\right) \cdot D^{2} \Phi\left(\xi_{i}\right)\left(\xi_{j}-\xi_{i}\right) \int_{\omega_{\xi_{i}}} D \lambda_{j} \cdot\left(D \lambda_{i}-\frac{\zeta}{h}\right) \\
-\Delta t \sum_{j \neq i} A_{i, j} F\left(D \Phi(\xi), D^{2} \Phi(\xi)\right)+O\left(h^{s+1}\right)
\end{array}\right) .
\end{aligned}
$$


Since $\Phi \in \mathrm{C}^{\infty}$ and since from (54), $\Delta t=o(h)$, we have that asymptotically for $h \rightarrow 0$ and $\Delta t \rightarrow 0$

$$
\begin{aligned}
0 & \geqslant-\mu\left|D \Phi\left(\xi_{i}\right)\right| \frac{\left|\omega_{\xi_{i}}\right|}{h}-\frac{1}{2} \sum_{j \neq i}\left(\xi_{j}-\xi_{i}\right) \cdot D^{2} \Phi\left(\xi_{i}\right)\left(\xi_{j}-\xi_{i}\right) \int_{\omega_{\xi_{i}}} D \lambda_{j} \cdot\left(D \lambda_{i}-\frac{\zeta}{h}\right) \\
& -\Delta t \sum_{j \neq i} A_{i, j} F\left(D \Phi(\xi), D^{2} \Phi(\xi)\right)+O\left(h^{s+1}\right),
\end{aligned}
$$

hence there exists a $\overline{\Delta t}>0$ such that for every $\Delta t<\overline{\Delta t}$ :

$$
u_{i}^{n+1} \leqslant \Phi\left(\xi_{i}\right)-\Delta t F\left(D \Phi(\xi), D^{2} \Phi(\xi)\right) .
$$

The scheme denoted by $\widetilde{S}$ in (49) may now be chosen as a generic scheme satisfying (32) at $\xi$. Since the test function $\Phi$ does not depend on time, $\widetilde{S}(\varphi ; i)$ verifies:

$$
\lim _{m \rightarrow \infty} \frac{\varphi\left(\xi_{i_{m}}\right)-\widetilde{S}\left(\varphi ; i_{m}\right)}{\Delta t_{m}}=F\left(D \Phi(\xi), D^{2} \Phi(\xi)\right),
$$

so that we have $\Phi\left(\xi_{i}\right)-\widetilde{S}(\varphi ; i)=\Delta t F\left(D \Phi(\xi), D^{2} \Phi(\xi)\right)+o(\Delta t)$ and we finally get

$$
u_{i}^{n+1} \leqslant \widetilde{S}(\varphi ; i)+o(\Delta t) \text {. }
$$

Case $2 \quad D \Phi(\xi)=0$.

When $\left|\left[D_{h} u\right]\left(\xi_{i}\right)\right| \leqslant C h^{s}$ the scheme is clearly monotone (in the conventional sense) at the node $\xi_{i}$, being a convex combination of node values.

For $\left|\left[D_{h} u\right]\left(\xi_{i}\right)\right| \geqslant C h^{s}$, the scheme still satisfies (44) since

$$
u_{i}^{n+1}=\widehat{S}\left(u^{n} ; i\right) \leqslant \widetilde{S}(\varphi ; i)
$$

where $\widetilde{S}(\varphi ; i)$ has been chosen in the following form,

$$
\begin{array}{r}
\widetilde{S}(\varphi ; i)=\Phi\left(\xi_{i}\right)+\frac{\Delta t}{\delta^{2}}\left(\frac{1}{2} \ell_{h}[\varphi]\left(\xi_{i}+\delta \Theta_{i}^{n}\right)+\frac{1}{2} \ell_{h}[\varphi]\left(\xi_{i}-\delta \Theta_{i}^{n}\right)-\Phi\left(\xi_{i}\right)\right) \\
-\frac{W \Delta t}{\delta h^{s+1}} \sum_{j} A_{i, j} \Phi\left(\xi_{j}\right),
\end{array}
$$

where $\Theta_{i}^{n}$ is given by (22), i.e., is computed from the values of $u^{n}$. The inequality (64) holds because the upwind points $\xi_{i} \pm \delta \Theta_{i}^{n}$ are the same on the left and right term and the time step $\Delta t$ verifies the second inequality in (54).

Moreover $\widetilde{S}$ is a consistent scheme, since under condition (42),

$$
\frac{\Phi\left(\xi_{i}\right)-\widetilde{S}(\varphi ; i)}{\Delta t}=\left(\Theta_{i}^{n}\right)^{T} D^{2} \Phi\left(\xi_{i}\right) \Theta_{i}^{n}+o(1) .
$$

Therefore, following the same arguments used in Case $2 a$ of the consistency proof,

$$
\begin{aligned}
\underline{F}\left(D \Phi(\xi), D^{2} \Phi(\xi)\right) & \leqslant \liminf _{m \rightarrow \infty} \frac{\Phi\left(\xi_{i_{m}}\right)-\widetilde{S}\left(\varphi ; i_{m}\right)}{\Delta t_{m}} \\
& \leqslant \limsup _{m \rightarrow \infty} \frac{\Phi\left(\xi_{i_{m}}\right)-\widetilde{S}\left(\varphi ; i_{m}\right)}{\Delta t_{m}} \leqslant \bar{F}\left(D \Phi(\xi), D^{2} \Phi(\xi)\right),
\end{aligned}
$$

which yields the consistency for $\widetilde{S}$ at $\xi$. 
Second inequality We have to check assumption (45). We now assume that, for $\Delta t \rightarrow 0$ and $\left(\xi_{i}, t_{n}\right) \rightarrow(x, t)$

$$
u_{i}^{n} \geqslant \Phi\left(\xi_{i}\right)
$$

We need to prove that

$$
\widehat{S}\left(u^{n} ; i\right) \geqslant \widetilde{S}(\varphi ; i)+o(\Delta t)
$$

in which the choice of $\widetilde{S}$ will follow the same guidelines used in proving (44).

Case $1 \quad D \Phi(\xi) \neq 0$.

As we have seen, for $h \rightarrow 0$, the condition $\left|D_{j}[\varphi]\right|>C h^{s}$ is asymptotically satisfied.

We consider the same subcases as before:

a) $\xi_{i}$ is a strongly internal node and $\left|\left[D_{h} u\right]\left(\xi_{i}\right)\right| \geqslant C h^{s}$. The result is obtained exactly as the first inequality in Case 1a).

b) $\xi_{i}$ is a strongly internal node and $\left|\left[D_{h} u\right]\left(\xi_{i}\right)\right| \leqslant C h^{s}$. In this case, we use the same argument as for the first inequality in Case 1b). Indeed, for any constant vector $\zeta$,

$$
\begin{aligned}
-\sum_{j \neq i} A_{i, j} u_{j} & =-\int_{\omega_{\xi_{i}}} D \ell_{h}[u] \cdot D \lambda_{i}+u_{i} D \lambda_{i} \cdot D \lambda_{i} \\
& =-\sum_{j \neq i} u_{j} \int_{\omega_{\xi_{i}}} D \lambda_{j} \cdot D \lambda_{i} \\
& \geqslant-\sum_{j \neq i} u_{j} \int_{\omega_{\xi_{i}}} D \lambda_{j} \cdot\left(D \lambda_{i}-\frac{\zeta}{h}\right)-C h^{s+1}
\end{aligned}
$$

and it is always possible to choose $\zeta$ small enough such that (57) holds true. With such a choice,

$$
\begin{aligned}
-\sum_{j \neq i} A_{i, j} u_{j} \geqslant & -\left(\sum_{j \neq i} A_{i, j}\right) \Phi\left(\xi_{i}\right)+\sum_{j \neq i}\left(\xi_{j}-\xi_{i}\right) \cdot D \Phi\left(\xi_{i}\right) \int_{\omega_{\xi_{i}}} D \lambda_{j} \cdot \frac{\zeta}{h} \\
& -\frac{1}{2} \sum_{j \neq i}\left(\xi_{j}-\xi_{i}\right) \cdot D^{2} \Phi\left(\xi_{i}\right)\left(\xi_{j}-\xi_{i}\right) \int_{\omega_{\xi_{i}}} D \lambda_{j} \cdot\left(D \lambda_{i}-\frac{\zeta}{h}\right)+O\left(h^{s+1}\right) .
\end{aligned}
$$

Hence, choosing now $\zeta=-\mu \frac{D \Phi\left(\xi_{i}\right)}{\left|D \Phi\left(\xi_{i}\right)\right|}$ with $\mu$ small enough such that (57) holds true one gets

$$
\begin{aligned}
-\sum_{j \neq i} A_{i, j} u_{j}^{n} \geqslant & -\left(\sum_{j \neq i} A_{i, j}\right) \Phi\left(\xi_{i}\right)+\mu\left|D \Phi\left(\xi_{i}\right)\right| \frac{\left|\omega_{\xi_{i}}\right|}{h} \\
& -\frac{1}{2} \sum_{j \neq i}\left(\xi_{j}-\xi_{i}\right) \cdot D^{2} \Phi\left(\xi_{i}\right)\left(\xi_{j}-\xi_{i}\right) \int_{\omega_{\xi_{i}}} D \lambda_{j} \cdot\left(D \lambda_{i}-\frac{\zeta}{h}\right)+O\left(h^{s+1}\right) .
\end{aligned}
$$

Since $u^{n}$ satisfies (24), we add and subtract $\Delta t F\left(D \Phi(\xi), D^{2} \Phi(\xi)\right)$ (note that $\underline{F}=\bar{F}=F$, since 
we are in the case $D \Phi(\xi) \neq 0)$, obtaining

$$
\begin{aligned}
& u_{j}^{n+1} \geqslant \Phi\left(\xi_{i}\right)-\Delta t F\left(D \Phi(\xi), D^{2} \Phi(\xi)\right) \\
& -\frac{1}{\sum_{j \neq i} A_{i, j}}\left(\begin{array}{l}
\mu\left|D \Phi\left(\xi_{i}\right)\right| \frac{\left|\omega_{\xi_{i}}\right|}{h}-\frac{1}{2} \sum_{j \neq i}\left(\xi_{j}-\xi_{i}\right) \cdot D^{2} \Phi\left(\xi_{i}\right)\left(\xi_{j}-\xi_{i}\right) \int_{\omega_{\xi_{i}}} D \lambda_{j} \cdot\left(D \lambda_{i}-\frac{\zeta}{h}\right) \\
-\Delta t \sum_{j \neq i} A_{i, j} F\left(D \Phi(\xi), D^{2} \Phi(\xi)\right)+O\left(h^{s+1}\right)
\end{array}\right),
\end{aligned}
$$

and as for the first inequality, there exists a $\overline{\Delta t}>0$ such that for every $\Delta t<\overline{\Delta t}$ :

$$
u_{i}^{n+1} \geqslant \Phi\left(\xi_{i}\right)-\Delta t F\left(D \Phi(\xi), D^{2} \Phi(\xi)\right) .
$$

We add and subtract a generic scheme $\widetilde{S}$ satisfying (32) and get

$$
u_{i}^{n+1} \geqslant \widetilde{S}(\varphi ; i)+o(\Delta t)
$$

Case $2: D \Phi(\xi)=0$.

If $\xi_{i}$ is a strongly internal node and $\left|\left[D_{h} u\right]\left(\xi_{i}\right)\right| \leqslant C h^{s}$, then we use the monotonicity of the scheme (24). Else, if $\xi_{i}$ is a strongly internal node and $\left|\left[D_{h} u\right]\left(\xi_{i}\right)\right|>C h^{s}$, we have

$$
u_{i}^{n+1}=\widehat{S}\left(u^{n} ; i\right) \geqslant \widetilde{S}(\varphi ; i),
$$

where $\widetilde{S}$ is the scheme chosen in the discussion of the first inequality, Case 2.

In conclusion, we have proved the following:

THEOREM 1 Let us suppose that Assumption 1 holds and that $\left[D_{h} u\right]$ is the first order approximation of the gradient defined in (19). Under the same assumptions as in Proposition 1 (with $q=1$ ), and under conditions (42) and (54), the regularized scheme (47) is monotone in the sense given by Definition 3.

COROLLARY 1 Under the assumptions of Theorem 1, the regularized scheme (47) is a convergent scheme for problem (1), (4), (5) when $h, \Delta t, \delta, h^{2} / \Delta t, h^{1-s} / \delta$ tend to zero.

Proof. The monotonicity of the regularized scheme comes from Theorem 1 . For the consistency, the same proof as that of Proposition 1 can be used, see Remark 7. The generalized Barles-Souganidis theorem can then be applied, thanks to the strong comparison principle stated in [3].

REMARK 8 It was shown in [7] that it was actually possible to find regimes in which the assumptions of Theorem 1 and of Corollary 1 hold, for example: $0<s<1, h=\delta^{\gamma}$, $\Delta t=\beta \delta^{1+\gamma(1+s)}$ where $\gamma$ is a parameter such that $\gamma(1-s)>1$ and $\beta$ is a positive number, small enough such that (54) holds.

REMARK 9 The analysis above has been made only in dimension two, for meshes with acute angles, in the case when $A$ is given by (10) and the interpolation operator is given by (18). In this case $A$ and the interpolation operator are closely related to each other and the proof takes advantage of this relationship. It would be very interesting to generalize the proof to cases when the two operators are more independent from each other, in particular for the scheme proposed in Remark 6, and also to dimension three. 


\section{Numerical results}

Let us examine some numerical tests in dimension two in order to verify the efficiency and accuracy of the approximation scheme described in $\S 2$ (note that we do not use the modified scheme mentioned in $\S 3.2$, which uses an artificial viscosity).

\subsection{An example proposed by G. Barles}

Take for $\Omega$ a ring with inner radius $r$ and outer radius $R>r$, and set $u_{0}(x)=\phi\left(|x|^{2}\right)$. G. Barles has proved in [3] that the viscosity solution of (1), (4), (5) is

$$
u(x, t)=\phi\left(\min \left(|x|^{2}+2 t, R^{2}\right)\right), \text { in } \bar{\Omega} \times(0,+\infty),
$$

for any value of $\theta \in(-1,1)$. The level set $u(x, t)=\phi\left(R^{2}\right)$ is the $\operatorname{ring} x \in\left[\max \left(\sqrt{R^{2}-2 t}, r\right), R\right]$. The partial differential equation holds up to the boundary $|x|=r$, and the boundary condition is lost there.

4.1.1 Results on a fully structured grid. Here, the domain is a ring with inner radius $r=0.1$ and outer radius $R=0.4$, and we have chosen $u_{0}(x)=16|x|^{2}$, so $u(x, t)=\min \left(16\left(|x|^{2}+2 t\right), 2.56\right)$. For a large integer $N$, the grid nodes are

$$
\xi_{i, j}=(r+i(R-r) / N)(\cos (2 j \pi / N), \sin (2 j \pi / N)), \quad i=0, \ldots, N, j=1, \ldots, N,
$$

and we take $\Delta t=(R-r) /(10 N)$ and $\delta=\sqrt{2 \Delta t}+2(R-r) / N$.

We have chosen $\theta=0.5$

On such a structured grid, the approximation of the gradient is a centered finite difference method in polar coordinates, so it is second order. Since the approximation of the gradient is second order, we choose $s=1$. In this case, it is convenient to replace (24) with an explicit Euler scheme for the equation

$$
\frac{\partial w}{\partial t}-\epsilon\left(\frac{\partial^{2} w}{\partial r^{2}}+\frac{\partial^{2} w}{\partial \theta^{2}}\right)=0
$$

with $\epsilon \sim \frac{1}{N^{2} \Delta t}$.

In this case, as seen in Table 1 , the error seems to decay like $N^{-\frac{1}{2}}$, which agrees with the estimates on the consistency error in (36).

TABLE 1. $\|$ Error $\|_{\infty}$

\begin{tabular}{lcccc}
\hline$N$ & 50 & 100 & 200 & 400 \\
\hline Error & 0.116 & 0.082 & 0.055 & 0.041 \\
Rel. Error & $4.53 \%$ & $3.2 \%$ & $2.14 \%$ & $1.6 \%$ \\
\hline
\end{tabular}

4.1.2 Results on a nonstructured grid. The domain is a ring with inner radius $r=1$ and outer radius $R=2$. We take $u^{0}(x)=|x|^{2}$, so the viscosity solution of (1), (4), (5) is $u(x, t)=\min \left(|x|^{2}+\right.$ $2 t, 4)$, for all $\theta$. 

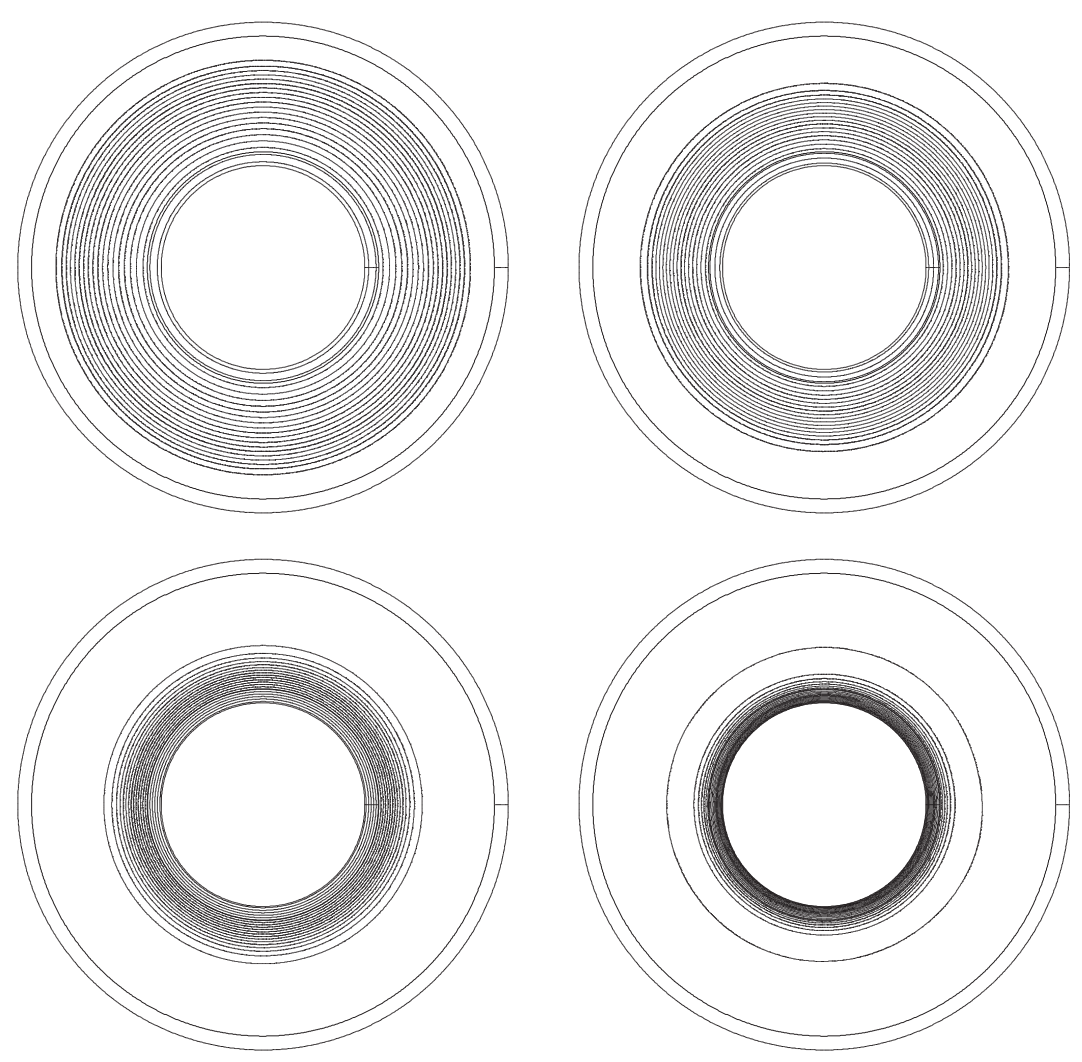

FIG. 4. $h=0.01$ : contour lines at $t=0.4,0.8,1.2,1.6$. The boundary zones are also presented in the figures.

For a given parameter $h$, we choose

$$
\Delta t=h / 10, \quad \delta=\sqrt{2 \Delta t}+2 h, \quad s=0.5 .
$$

We have chosen $\theta=-0.5$. Table 2 contains the errors in maximum norm with respect to the explicit solution for different vales of $h$. It can be seen that the error decays to zero like $\sqrt{h}$. The contour lines of the solution at different times are shown in Figure 4, along with the two circles representing the boundaries of the layers $\omega^{1}$ and $\omega^{2}$. We see that the scheme captures correctly the zones where the solution is constant. The error in maximum norm with respect to the explicit solution as a function of time is plotted in Figure 5.

TABLE 2. $\|$ Error $\|_{\infty}$

\begin{tabular}{lcccc}
\hline$h$ & 0.04 & 0.02 & 0.01 & 0.0064 \\
\hline Error & 0.21 & 0.154 & 0.114 & 0.095 \\
Rel. Error & $5.3 \%$ & $3.8 \%$ & $2.85 \%$ & $2.37 \%$ \\
\hline
\end{tabular}




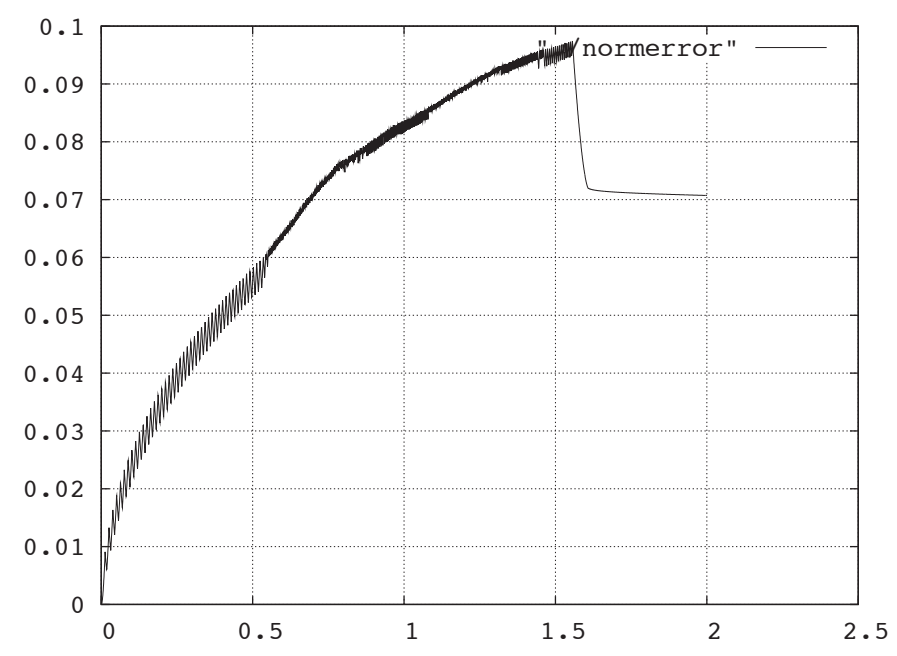

FIG. 5. $h=0.0064$ : error in max norm vs. time

\subsection{Other examples}

4.2.1 Test 2. Here, the domain is a ring with inner radius $r=1$ and outer radius $R=2$, and we take $u_{0}(x)=\sin \left(\frac{16}{25}\left((x-0.5)^{2}+x_{2}^{2}\right)\right)$. We use an unstructured mesh such that $h \sim 0.02$ and take $\Delta t=0.002$. The thickness of the layers near the boundaries is of the order of 0.2.

The contour lines at different times, for $\theta=-0.5$, (resp. $\theta=0.95$ ) are displayed in Figure 6 (resp. 7). In the first (resp. second) case the contour lines make an angle of $30^{\circ}\left(\right.$ resp $\sim 71.8^{\circ}$ ) with the normal to the boundary.

4.2.2 Test 3. The domain is constructed as follows. It has a hole. The outer boundary is parametrized by

$$
x_{1}=2 \cos (2 \pi t)+0.75 \cos (4 \pi t), \quad x_{2}=2 \sin (2 \pi t)+0.75 \sin (4 \pi t),
$$

and the inner boundary is the unit circle of equation $|x-(0.5,0)|=1$. We choose $\theta=-0.5$ and $u^{0}(x)=\left|x_{1}\right| / 10$. We take

$$
h=0.02, \quad \delta=0.1, \quad \Delta t=0.001 .
$$

Contour lines are displayed in Figure 8. We see that a level set with non empty interior appears.

4.2.3 Test 4. The domain is constructed as follows. It has a hole. The outer boundary is parametrized by

$$
x_{1}=2 \cos (2 \pi t)+0.75 \cos (4 \pi t), \quad x_{2}=2 \sin (2 \pi t)+0.75 \sin (4 \pi t),
$$

and the inner boundary is the unit circle centered at 0 . We choose $\theta=-0.5$ and $u^{0}(x)=\sin (4|x|)$. We take an unstructured mesh with

$$
h=0.02, \quad \delta=0.1, \quad \Delta t=0.001 .
$$

Contour lines are displayed in Figure 9. 

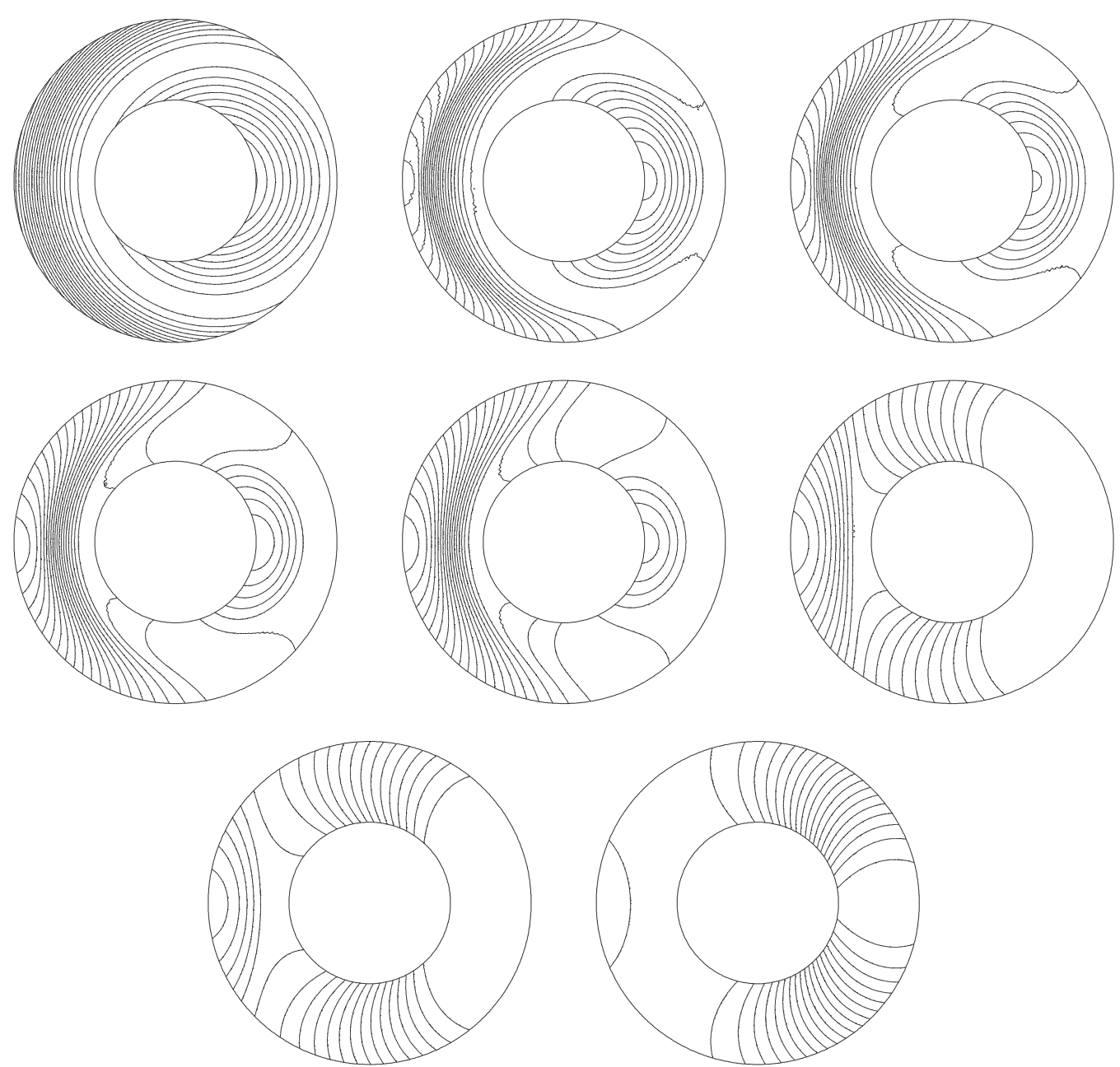

FIG.6. Test 2: $\theta=-0.5$, contour lines at $t=0,0.08,0.16,0.24,0.32,0.8,1.2,2$ 

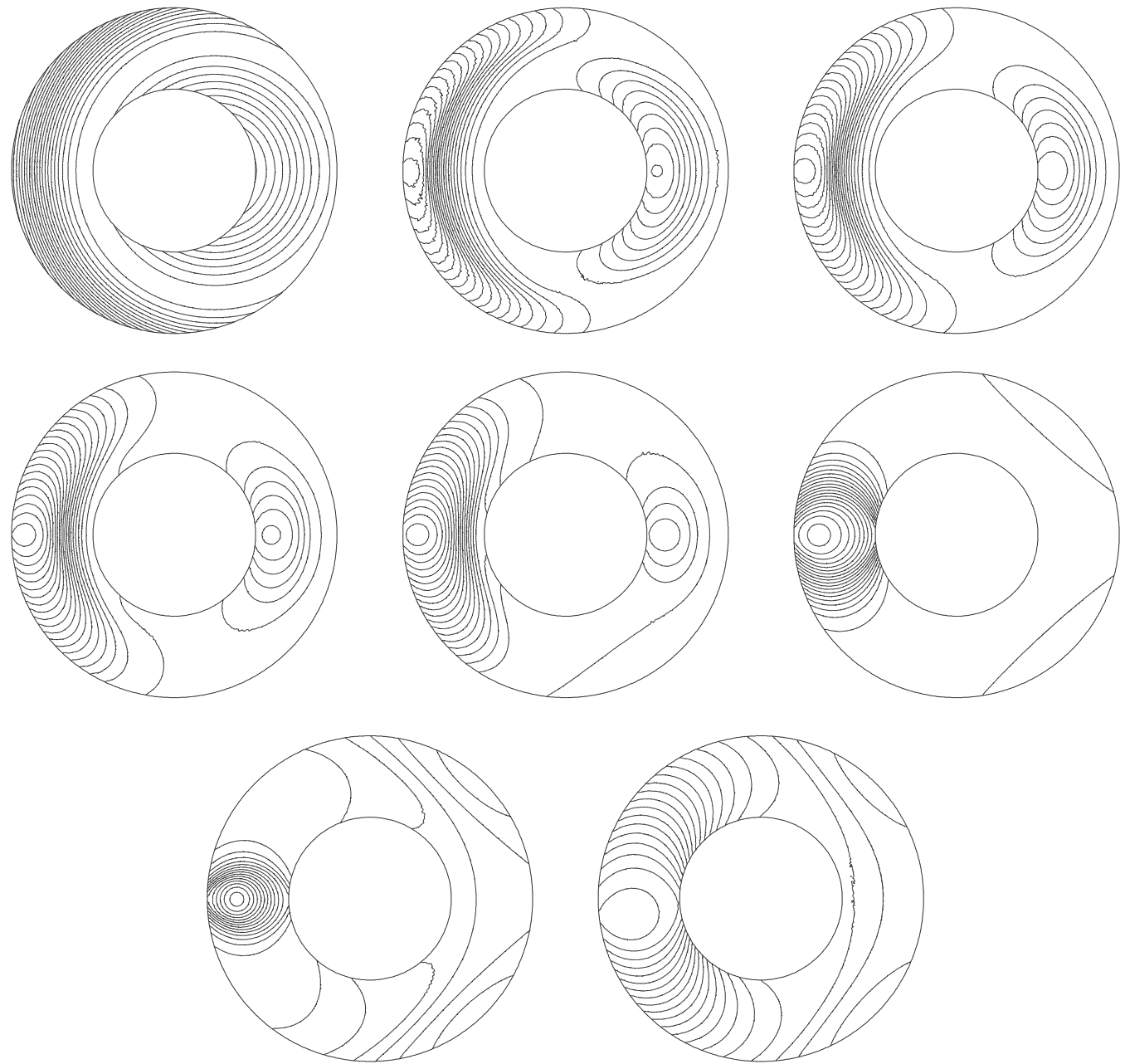

FIG. 7. Test 2: $\theta=0.95$, contour lines at $t=0,0.08,0.16,0.24,0.32,0.8,1.2,2$ 
482
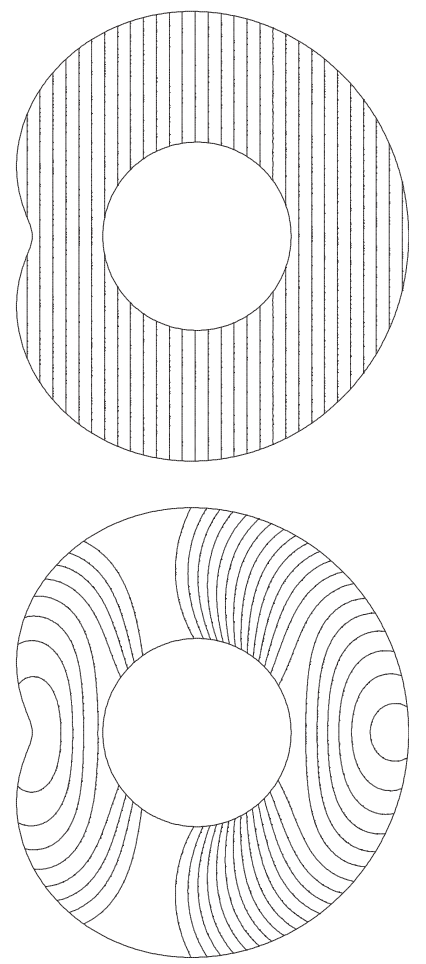

Y. ACHDOU AND M. FALCONE
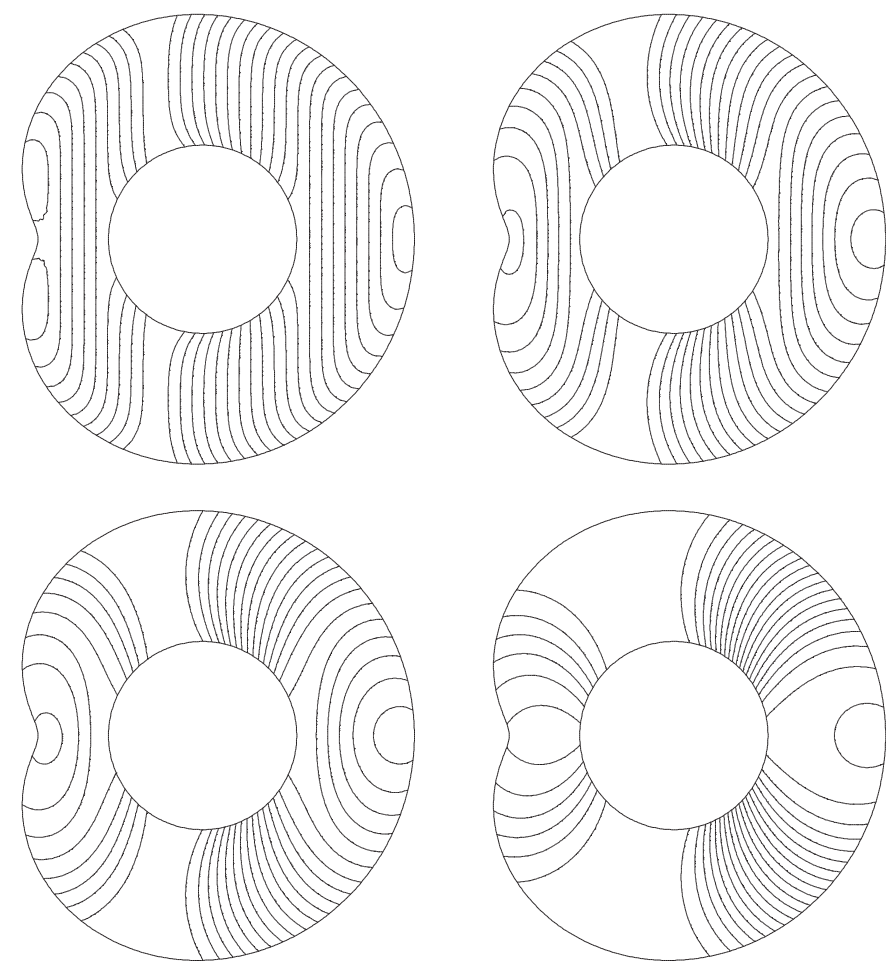
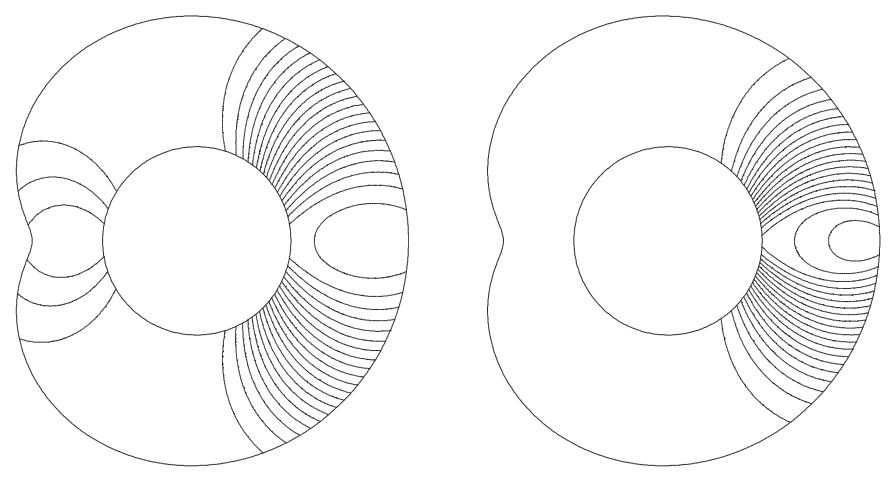

FIG. 8. Test 3: Contour lines at $t=0,0.08,0.16,0.24,0.32,0.8,1.2,2$ 

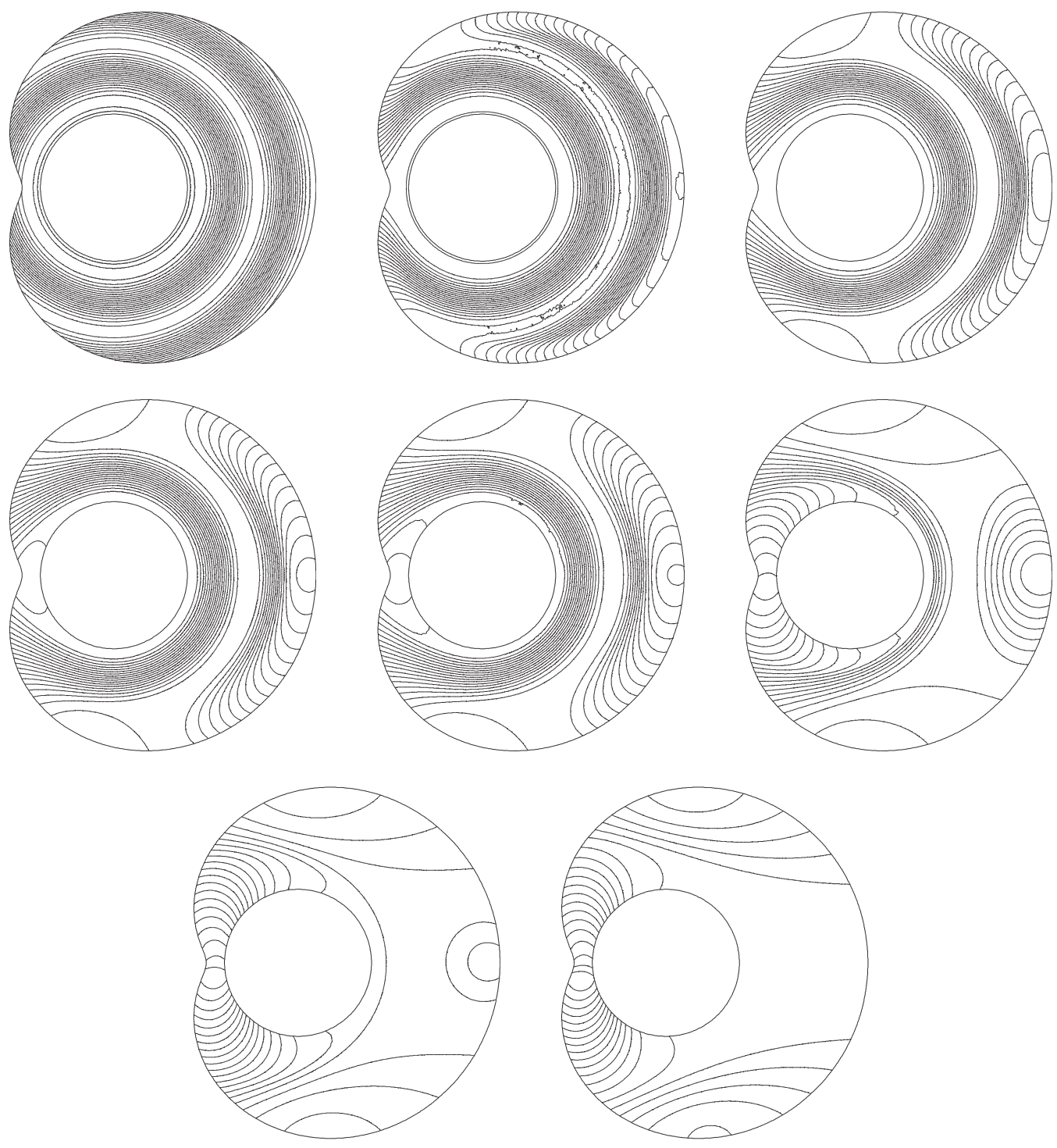

FIG.9. Test 4: Contour lines at $t=0,0.08,0.16,0.24,0.32,0.8,1.2,2$

\section{REFERENCES}

1. AbGRall, R., Numerical discretization of the first-order Hamilton-Jacobi equation on triangular meshes, Comm. Pure Appl. Math. 49 (1996), 1339-1373. Zbl0870.65116 MR1414589b6

2. BARles, G., Solutions de viscosité des équations de Hamilton-Jacobi, Mathématiques \& Applications (Berlin) [Mathematics \& Applications], vol. 17, Springer-Verlag, Paris, 1994. Zbl0819.35002 MR1613876 
3. BARLES, G., Nonlinear Neumann boundary conditions for quasilinear degenerate elliptic equations and applications, J. Differential Equations 154 (1999), 191-224. Zbl0924 . 35051 MR1685618

4. BARles, G., \& GeORgelin, C., A simple proof of convergence for an approximation scheme for computing motions by mean curvature, SIAM J. Numer. Anal. 32 (1995), 484-500. Zb10831.65138 MR1324298

5. Barles, G., \& Souganidis, P. E., Convergence of approximation schemes for fully nonlinear second order equations, Asymptotic Anal. 4 (1991), 271-283. Zb10729.65077 MR1115933

6. Buckdahn, R., Cardaliaguet, P., \& Quincampoix, M., A representation formula for the mean curvature motion, SIAM J. Math. Anal. 33 (2001), 827-846 (electronic). Zbl1074. 93037 MR1884724

7. Carlini, E., Falcone, M., \& Ferretti, R., Convergence of a large time-step scheme for mean curvature motion, Interfaces Free Bound., 12 (2010), 409-441. Zblpre05864089 MR2754211

8. Catté, F. Dibos, F., \& Koepfler, G., A morphological scheme for mean curvature motion and applications to anisotropic diffusion and motion of level sets, SIAM J. Numer. Anal. 32 (1995), 1895-1909. Zb10841.68124 MR1360464

9. Chen, Y. G., Giga, Y., \& Goto, S., Uniqueness and existence of viscosity solutions of generalized mean curvature flow equations, J. Differential Geom. 33 (1991), 749-786. Zb10696. 35087 MR1100211

10. Cockburn, B., \& QIAN, J., Continuous dependence results for Hamilton-Jacobi equations, Collected lectures on the preservation of stability under discretization (Fort Collins, CO, 2001), SIAM, Philadelphia, PA, 2002, pp. 67-90. MRMR2026664

11. Crandall, M. G., Ishit, H., \& LiOns, P.-L., User's guide to viscosity solutions of second order partial differential equations, Bull. Amer. Math. Soc. (N.S.) 27 (1992), 1-67. Zb10755. 35015 MR1118699

12. Crandall, M. G., \& Lions, P.-L., Two approximations of solutions of Hamilton-Jacobi equations, Math. Comp. 43 (1984), 1-19. Zbl0556.65076 MR0744921

13. Crandall, M. G., \& Lions, P.-L., Convergent difference schemes for nonlinear parabolic equations and mean curvature motion, Numer. Math. 75 (1996), 17-41. Zbl0874. 65066 MR1417861

14. Deckelnick, K., \& DZIUK, G., A fully discrete numerical scheme for weighted mean curvature flow, Numer. Math. 91 (2002), 423-452. Zb10999.65103 MR1907866

15. Evans, L. C., Convergence of an algorithm for mean curvature motion, Indiana Univ. Math. J. 42 (1993), 533-557. Zbl0802.65098 MR1237058

16. Evans, L. C., \& Spruck, J., Motion of level sets by mean curvature. I, J. Differential Geom. 33 (1991), 635-681. Zbl0726.53029 MR1100206

17. Falcone, M., \& Ferretti, R., Semi-Lagrangian schemes for Hamilton-Jacobi equations, discrete representation formulae and Godunov methods, J. Comput. Phys. 175 (2002), 559-575. Zbl1007.65060 MR1880118

18. Falcone, M., \& Ferretti, R., Consistency of a large time-step scheme for mean curvature motion, Numerical mathematics and advanced applications, Springer Italia, Milan, 2003, pp. 495-502. Zb11045. 65072 MR2360748

19. Giga, Y., Surface evolution equations, Monographs in Mathematics, vol. 99, Birkhäuser Verlag, Basel, 2006, A level set approach. Zbl1096.53039 MR2238463

20. Giga, Y., Ohnuma, M., \& SAto, M.-H., On the strong maximum principle and the large time behavior of generalized mean curvature flow with the Neumann boundary condition, J. Differential Equations 154 (1999), 107-131. Zbl0930.35029 MR1685634

21. IsHII, H., \& ISHII, K., An approximation scheme for motion by mean curvature with right-angle boundary condition, SIAM J. Math. Anal. 33 (2001), 369-389 (electronic). Zb10994. 35012 MR1857975

22. Kohn, R. V., \& Serfaty, S., A deterministic-control-based approach to motion by curvature, Comm. Pure Appl. Math. 59 (2006), 344-407. Zbl1206.53072 MR2200259

23. Nochetto, R. H., \& Verdi, C., Convergence past singularities for a fully discrete approximation of curvature-driven interfaces, SIAM J. Numer. Anal. 34 (1997), 490-512. Zb10876. 35053 MR1442924 
24. Oberman, A., A convergent monotone difference scheme for motion of level sets by mean curvature, Numer. Math. 99 (2004), 365-379. Zbl1070.65082 MR2107436

25. Osher, S., \& FEDKIW, R., Level set methods and dynamic implicit surfaces, Applied Mathematical Sciences, vol. 153, Springer-Verlag, New York, 2003. Zbl1026.76001 MR1939127

26. Osher, S., \& Sethian, J. A., Fronts propagating with curvature-dependent speed: algorithms based on Hamilton-Jacobi formulations, J. Comput. Phys. 79 (1988), 12-49. Zbl0659. 65132 MR0965860

27. Sagona, M., \& Seghini, A., An adaptive scheme on unstructured grids for the shape-from-shading problem, Numerical methods for viscosity solutions and applications (Heraklion, 1999), Ser. Adv. Math. Appl. Sci., vol. 59, World Sci. Publ., River Edge, NJ, 2001, pp. 197-219. Zb10988.65101 MR1886714

28. SethiAn, J. A., Level set methods and fast marching methods, second ed., Cambridge Monographs on Applied and Computational Mathematics, vol. 3, Cambridge University Press, Cambridge, 1999, Evolving interfaces in computational geometry, fluid mechanics, computer vision, and materials science. Zbl0973. 76003 MR1700751

29. Soner, H. M., \& TOUZI, N., Dynamic programming for stochastic target problems and geometric flows, J. Eur. Math. Soc. (JEMS) 4 (2002), 201-236. Zbl1003. 49003 MR1924400

30. SONER, H. M., \& TouZI, N., A stochastic representation for mean curvature type geometric flows, Ann. Probab. 31 (2003), 1145-1165. Zbl1080.60076 MR1988466

31. Souganidis, P., Front propagation: theory and applications, Viscosity solutions and applications (Montecatini Terme, 1995), Lecture Notes in Math., vol. 1660, Springer, Berlin, 1997, pp. 186-242. Zb10882.35016 MR1462703

32. ZhaO, H., A fast sweeping method for eikonal equations, Math. Comp. 74 (2005), 603-627 (electronic). Zbl1070.65113 MR2114640 